\title{
Combinatorial Structure of Schulte's Chiral Polyhedra
}

\author{
Daniel Pellicer • Asia Ivić Weiss
}

Dedicated to Tomaž Pisanski on the occasion of his 60th birthday.

Received: 7 December 2008 / Revised: 6 July 2009 / Accepted: 25 December 2009 /

Published online: 18 February 2010

(C) Springer Science+Business Media, LLC 2010

\begin{abstract}
Schulte classified the discrete chiral polyhedra in Euclidean 3-space and showed that they belong to six families. The polyhedra in three of the families have finite faces and the other three families consist of polyhedra with (infinite) helical faces. We show that all the chiral polyhedra with finite faces are combinatorially chiral. However, the chiral polyhedra with helical faces are combinatorially regular. Moreover, any two such polyhedra with helical faces in the same family are isomorphic.
\end{abstract}

Keywords Combinatorially chiral · Geometrically chiral · Chiral polyhedron · Geometric polyhedron

\section{Introduction}

The main object of this paper is to examine chiral polyhedra in Euclidean 3-space from both abstract and geometric point of view. The term "chiral" has been used for geometric figures which are symmetrical by rotations but not by reflections. An example of such object is the snub cube. However, the term chiral map (or abstract chiral polyhedron, as we shall prefer to call them) is commonly used to describe a map on a surface whose symmetry group contains all cyclic permutations of consecutive edges through any vertex and of consecutive edges on any face. Many examples, including infinite families, of chiral finite (abstract) polyhedra are known to exist (see $[1,2,4])$. This naturally leads to the question whether there are any infinite chiral (abstract) polyhedra which we answer in affirmative in Corollary 6.1.

We start with a brief historical note on geometric regular and chiral polyhedra in Euclidean 3-space.

Supported by NSERC of Canada Grant \#8857.

D. Pellicer $(\bowtie) \cdot$ A. Ivić Weiss

York University, Toronto, Ontario M3J 1P3, Canada

e-mail:dpellicer@matem.unam.mx 
A polyhedron is geometrically regular if its geometric symmetry group is transitive on its flags. The only finite regular polyhedra in $\mathbb{E}^{3}$ are the platonic solids, the Kepler-Poinsot polyhedra and their Petrials (see [8, Theorem 7E4]). In 1926, Petrie and Coxeter discovered the three discrete infinite regular polyhedra in $\mathbb{E}^{3}$ with convex faces (see [3]). Grünbaum was the first to attempt the classification of infinite, nonplanar regular polyhedra with non-planar polygons or apeirogons (infinite polygons) as faces (see [7]). He listed 23 such polyhedra and the classification was completed by Dress (see $[5,6]$ ) by addition of a single polyhedron.

A polyhedron is geometrically chiral if its geometric symmetry group has precisely two orbits on the flags such that adjacent flags are in distinct orbits. In [10] and [11], Schulte enumerates the discrete chiral polyhedra in $\mathbb{E}^{3}$. In [10], he shows that no geometric chiral polyhedron in $\mathbb{E}^{3}$ can be finite and determines all infinite polyhedra with finite (skew) faces. He also shows that such infinite polyhedra belong to three families of polyhedra of Schläfli types $\{6,6\},\{4,6\}$ and $\{6,4\}$. In [11], he completes the enumeration by showing that there are no additional chiral polyhedra with finite faces and that any chiral polyhedron with infinite (helical) faces must be in one of three families, two of type $\{\infty, 3\}$ and one of type $\{\infty, 4\}$.

The main purpose of this paper is to investigate the combinatorial structure of Schulte's geometrically chiral polyhedra in $\mathbb{E}^{3}$. For each such polyhedron, we provide a presentation of the automorphism group. In Sect. 4, we prove that every such polyhedron with finite faces is combinatorially chiral. Schulte's chiral polyhedra with infinite faces are shown in Sect. 5 to be combinatorially regular. Furthermore, any two chiral polyhedra with infinite faces in the same family are isomorphic. We provide both, an algebraic proof of this fact as well as a geometric interpretation of the result. We show that the vertices and edges of any geometric chiral polyhedron in a family can be moved continuously to coincide with the vertices and edges of any other chiral polyhedron in the same family.

\section{Regular and Chiral Polyhedra and Their Realizations}

We begin by briefly outlining some basic theory of abstract polytopes of rank 3 referring to [8] and [12] for more details.

An abstract polygon $\mathcal{P}$ is a connected (may be infinite) graph with the property that every vertex is contained in exactly two edges.

An abstract polyhedron $\mathcal{K}$ is a partially ordered set satisfying the following conditions. The maximal totally ordered subsets of $\mathcal{K}$ (also called flags) contain exactly three elements. This induces a rank function from the elements of $\mathcal{K}$ to the set $\{0,1,2\}$. The elements of rank 0 are called vertices, the elements of rank 1 are called edges, and the elements of rank 2 are called faces. The vertex figure at a vertex $v$ consists of the edges and faces containing $v$. Note that the vertex and edge set of any polyhedron $\mathcal{K}$ naturally induces a graph, called the 1 -skeleton of $\mathcal{K}$. For $\mathcal{K}$ to be a polyhedron we require that every edge of $\mathcal{K}$ contains exactly two vertices and to be contained in exactly two faces. The 1 -skeleton of any polyhedron $\mathcal{K}$ must be connected. We also require that (the section consisting of every vertex and edge contained in) every face and every vertex-figure of $\mathcal{K}$ is isomorphic to a polygon. A more 
general definition of abstract polytope can be found in [8, Chap. 2A]. In the notation of [8], polygons are 2-polytopes and polyhedra are 3-polytopes.

As a consequence of the definition of abstract polyhedron, for any flag $\Phi$ there exist three distinguished flags: the 0 -adjacent flag obtained from $\Phi$ by preserving the edge and face but changing the vertex, the 1-adjacent flag obtained by preserving the vertex and the face but changing the edge, and the 2-adjacent flag obtained by preserving the vertex and the edge but changing the face.

The dual of a polyhedron $\mathcal{K}$ is the polyhedron obtained by preserving the elements of $\mathcal{K}$ while reversing the partial order. A polyhedron is said to be self-dual if it is isomorphic to its dual.

Whenever a polyhedron $\mathcal{K}$ has the property that every face contains the same number $p$ of edges and every vertex is contained in the same number $q$ of edges we say that $\mathcal{K}$ is equivelar. In this case, we say that the Schläfli type of the polyhedron is $\{p, q\}$. Regular and chiral polyhedra defined below are examples of equivelar polyhedra. In general, $p$ and $q$ may be $\infty$; however, in this paper $q$ will always be finite.

An automorphism of a polyhedron $\mathcal{K}$ is an order preserving permutation of its elements. The group consisting of all the automorphisms of $\mathcal{K}$, called the automorphism group of $\mathcal{K}$, is denoted by $\Gamma(\mathcal{K})$.

A polyhedron $\mathcal{K}$ is said to be regular if its automorphism group $\Gamma(\mathcal{K})$ is transitive on its flags. In this case, for any given base flag $\Phi$, there are three special involutory automorphisms $\rho_{0}, \rho_{1}$ and $\rho_{2}$ that map $\Phi$ into its 0 -, 1- and 2-adjacent flag, respectively. These involutions generate $\Gamma(\mathcal{K})$ and satisfy at least the relations

$$
\rho_{i}^{2}=\left(\rho_{0} \rho_{2}\right)^{2}=\left(\rho_{0} \rho_{1}\right)^{p}=\left(\rho_{1} \rho_{2}\right)^{q}=\varepsilon,
$$

as well as the intersection condition given by $\left\langle\rho_{0}, \rho_{1}\right\rangle \cap\left\langle\rho_{1}, \rho_{2}\right\rangle=\left\langle\rho_{1}\right\rangle$. Note that if $p$ is $\infty$, the relation $\left(\rho_{0} \rho_{1}\right)^{p}=\varepsilon$ is omitted.

Whenever the relations in (1) completely determine $\Gamma(\mathcal{K})$ we simply denote $\mathcal{K}$ by $\{p, q\}$. In this case, we refer to $\mathcal{K}$ as the universal (regular) polyhedron of Schläfli type $\{p, q\}$, and to its automorphism group as the Coxeter group $[p, q]$.

Given a regular polyhedron $\mathcal{K}$ with automorphism group $\Gamma(\mathcal{K})=\left\langle\rho_{0}, \rho_{1}, \rho_{2}\right\rangle$ we define two special automorphisms which we shall call distinguished rotations $\sigma_{1}:=\rho_{0} \rho_{1}$ and $\sigma_{2}:=\rho_{1} \rho_{2}$. Note that these automorphisms are not necessarily geometric rotations. The rotations $\sigma_{1}, \sigma_{2}$ generate the even subgroup $\Gamma^{+}(\mathcal{K})$ of $\Gamma(\mathcal{K})$ and satisfy at least the relations

$$
\sigma_{1}^{p}=\sigma_{2}^{q}=\left(\sigma_{1} \sigma_{2}\right)^{2}=\varepsilon,
$$

and the intersection condition

$$
\left\langle\sigma_{1}\right\rangle \cap\left\langle\sigma_{2}\right\rangle=\{\varepsilon\} .
$$

Similarly as above, if $p=\infty$ then the relation $\sigma_{1}^{p}=\varepsilon$ is omitted. Note that the index of $\Gamma^{+}(\mathcal{K})$ in $\Gamma(\mathcal{K})$ is at most 2. If this index is 2 , we say that $\mathcal{K}$ is orientably regular or, as in the language of [8] and [12] directly regular, otherwise we say that $\mathcal{K}$ is non-orientably regular.

The even subgroup of the automorphism group of the universal polyhedron $\{p, q\}$ is denoted by $[p, q]^{+}$. 
A polyhedron $\mathcal{K}$ is said to be chiral whenever there are two orbits of flags under $\Gamma(\mathcal{K})$ such that adjacent flags belong to different orbits. Choosing a base flag $\Phi$, the automorphism group of a chiral polyhedron $\mathcal{K}$ is generated by two automorphisms $\sigma_{1}$ and $\sigma_{2}$, which will also be referred to as distinguished rotations, that cyclically permute the edges of the face in $\Phi$ and the edges around the vertex in $\Phi$, respectively. These generators satisfy at least the relations (2) as well as the intersection condition given by (3). Each chiral polyhedron occurs in two enantiomorphic forms, in a sense in a right and left handed version which can be thought of as mirror images of each other. For details we refer to [13].

Note that the dual of a regular or chiral polyhedron of type $\{p, q\}$ is a regular or chiral polyhedron of type $\{q, p\}$.

In [12], Schulte and the second author provide a construction of chiral or orientably regular abstract polytopes from groups generated by "rotations" satisfying an intersection condition (above stated for rank 3 ). The polyhedra so constructed are shown to be regular if an only if there exists an involutory group automorphism $\hat{\rho}_{1}$ such that

$$
\sigma_{1} \hat{\rho}_{1}=\sigma_{1}^{-1} \text { and } \sigma_{2} \hat{\rho}_{1}=\sigma_{2}^{-1} .
$$

If $\mathcal{K}$ is non-orientably regular then the distinguished generators of its automorphism group satisfy (2), (3) and $\Gamma(\mathcal{K})$ coincides with its even subgroup. The above construction can then be used to construct a directly regular polyhedron, called the orientably regular double cover of $\mathcal{K}$, whose even subgroup coincides with that of $\mathcal{K}$. This implies the following two lemmas.

Lemma 2.1 Every non-orientably regular polyhedron has a directly regular double cover.

Lemma 2.2 Let $\mathcal{K}$ and $\mathcal{P}$ be chiral or orientably regular polyhedra. If the subgroups of the automorphism groups of $\mathcal{K}$ and $\mathcal{P}$ generated by the distinguished rotations $\sigma_{1}$ and $\sigma_{2}$ are isomorphic, then $\mathcal{P} \cong \mathcal{K}$. In particular, they are both chiral or both orientably regular.

Following [8, Sect. 5A], [10] and [11], a realization of an abstract polyhedron $\mathcal{K}$ in Euclidean 3-space is a mapping $\beta=\beta_{0}$ from the vertex set of $\mathcal{K}$ into $\mathbb{E}^{3}$. This mapping induces two other mappings $\beta_{1}$ and $\beta_{2}$ from the sets of edges and faces of $\mathcal{K}$ to the power set of the vertex set and the power set of the edge set of $\mathcal{K}$ respectively, such that for each edge $e_{0}$ and face $f_{0}$ of $\mathcal{K}$,

$$
\begin{aligned}
& e_{0} \beta_{1}=\left\{v \beta_{0} \mid v \subseteq e_{0}\right\}, \\
& f_{0} \beta_{2}=\left\{e \beta_{1} \mid e \subseteq f_{0}, e \text { edge of } \mathcal{K}\right\} .
\end{aligned}
$$

We say that a realization is faithful if $\beta_{0}, \beta_{1}$ and $\beta_{2}$ are one-to-one. In this paper, we mainly consider discrete faithful realizations of polyhedra having the property that the affine hull of their vertices is $\mathbb{E}^{3}$. The only exceptions are some discrete (but not faithful) realizations included in Sect. 5 for sake of completeness. Henceforth, unless otherwise stated, we shall assume that the affine hull of the vertex set of a geometric polyhedron is $\mathbb{E}^{3}$. 
When $\beta$ is a faithful realization of $\mathcal{K}$, the vertices, edges and faces of $\mathcal{K}$ are in bijective correspondence with some points, line segments and simple (finite or infinite) polygons in $\mathbb{E}^{3}$. The resulting set of points, line segments and polygons constitute the vertices, edges and faces of a polyhedron in $\mathbb{E}^{3}$. When there is no possibility of confusion we may identify an abstract polyhedron $\mathcal{K}$ with its (faithful) realization and call it geometric polyhedron $\mathcal{K}$.

A symmetry of the geometric polyhedron $\mathcal{K}$ is an isometry of $\mathbb{E}^{3}$ that preserves $\mathcal{K}$. The symmetry groups of the polyhedra we will consider in this paper are all (affinely) irreducible meaning that there is no non-trivial linear subspace $L$ of $\mathbb{E}^{3}$ which is invariant in the sense that the group permutes the set of translates of $L$ (see [8, Chap. 5A]).

A realization of a polyhedron $\mathcal{K}$ is said to be symmetric if each automorphism of $\mathcal{K}$ induces an isometric permutation of its set of vertices. Such a permutation can be extended to an isometry of $\mathbb{E}^{3}$ (whenever the affine hull of the set of vertices is $\mathbb{E}^{3}$ ). Note that if a realization of a polyhedron $\mathcal{K}$ is not symmetric, then the symmetry group of $\mathcal{K}$ is isomorphic to a proper subgroup of $\Gamma(\mathcal{K})$.

A geometric polyhedron is said to be geometrically regular if its symmetry group is transitive on its flags. In this case, the symmetry group is isomorphic to the automorphism group implying that the polyhedron is also regular as an abstract polyhedron. In [8, Sect. 7E], McMullen and Schulte provide a survey of all the geometrically regular polyhedra (Grünbaum-Dress polyhedra) in $\mathbb{E}^{3}$. There are 24 infinite geometrically regular polyhedra such that the affine hull of their vertices is $\mathbb{E}^{3}$. They are divided in two families, 12 blended polyhedra having reducible symmetry groups (the groups permutes a linear subspace of $\mathbb{E}^{3}$ and its translates) and 12 pure polyhedra having irreducible symmetry groups.

A geometric polyhedron $\mathcal{K}$ arising from a regular or chiral abstract polyhedron is said to be geometrically chiral if its symmetry group has two orbits on its flags in such a way that adjacent flags are in different orbits. The symmetry group of a geometrically chiral polyhedron can be generated by two isometries $S_{1}$ and $S_{2}$ that correspond to the distinguished rotations $\sigma_{1}$ and $\sigma_{2}$ in $\Gamma(\mathcal{K})$, respectively. The symmetry group of any geometrically chiral polyhedron $\mathcal{K}$ is isomorphic to a subgroup of the automorphism group of $\mathcal{K}$ of index at most 2 . When the index is $2, \mathcal{K}$ must necessarily be regular as an abstract polyhedron and the automorphisms represented by the symmetries are precisely the elements of $\Gamma^{+}(\mathcal{K})$. We also say that $\mathcal{K}$ admits a chiral realization. On the other hand, when the index is $1, \mathcal{K}$ is chiral as an abstract polyhedron. In other words, if a polyhedron admits a chiral realization it must necessarily be either chiral or orientably regular. This directly implies

Lemma 2.3 Let $\mathcal{K}$ and $\mathcal{P}$ be two polyhedra admitting chiral realizations. If the subgroups of the symmetry groups of $\mathcal{K}$ and $\mathcal{P}$ generated by the distinguished rotations $S_{1}$ and $S_{2}$ are isomorphic, then $\mathcal{P} \cong \mathcal{K}$. In particular, they are both chiral or both orientably regular as abstract polytopes.

In [10] and [11], Schulte classifies all the geometrically chiral polyhedra in $\mathbb{E}^{3}$; however, he did not determine whether or not they are abstractly chiral. We shall do this in Sects. 4 and 5. 
Note that the isometries $S_{i}, i=1,2$, can be either rotations, rotatory reflections, or, as we shall see in Sect. 5, $S_{1}$ (but not $S_{2}$ ) can also be a twist. Each $S_{i}$ is therefore a product of a $r_{i}$-fold rotation $R_{i}$ and an isometry $T_{i}$ where $T_{i}$ is either $\varepsilon$, a reflection with mirror orthogonal to the axis of $R_{i}$, or a translation by a vector parallel to the axis of $R_{i}$. If $T_{1}=\varepsilon$ (resp., $T_{2}=\varepsilon$ ), the faces (resp., vertex-figures) of $\mathcal{K}$ are regular convex polygons. If $T_{1}$ (resp., $T_{2}$ ) is a reflection then it was proved in [10] that $r_{i}$ must be 4 or 6 and the faces (resp., vertex-figures) are skew $r_{i}$-gons. If the angle of the rotation $R_{i}$ is $2 \pi / r_{i}$ and $T_{i}$ is either $\varepsilon$ or a reflection, the Schläfli symbol for $\mathcal{K}$ is $\left\{r_{1}, r_{2}\right\}$. If $T_{1}$ is a twist, the faces of $\mathcal{K}$ are helices and, as proved in [11], $r_{1}$ must be either 3 or 4 and we shall say that the helical faces are over triangles or over squares, respectively. In this case, the Schläfli type for $\mathcal{K}$ is $\left\{\infty, r_{2}\right\}$. When $r_{2}=3$ the helices can be over triangles or over squares. When $r_{2}=4$ the helices must be over squares.

In conclusion of this section, we provide some useful definitions regarding words on the generators of the symmetry group of a geometrically chiral polyhedron, or of the even subgroup of the symmetry of a geometrically regular polyhedron.

Let $G=\left\langle S_{1}, S_{2}\right\rangle$ be the rotation group of a geometric chiral or regular polyhedron and $w=S_{i_{1}} S_{i_{2}} \cdots S_{i_{k}}$ a word in the generators of $G$. Let $w^{*}=S_{i_{1}}^{-1} S_{i_{2}}^{-1} \cdots S_{i_{k}}^{-1}$. We shall call $w^{*}$ the enantiomorphic word corresponding to $w$.

Note that for any word $w$ corresponding to the symmetry $S$ in $G, w^{*}$ can also be seen to correspond to a symmetry $S^{\prime}$ of the polyhedron. Moreover, as a consequence of (4), if the polyhedron is regular, then $S^{\prime}$ depends only on $S$ and not of the word defining it. In this case, we say that $S^{\prime}$ is the enantiomorphic element of $S$.

\section{Preliminary Results on Isometries in $\mathbb{E}^{3}$}

The discrete chiral polyhedra in $\mathbb{E}^{3}$ must have irreducible symmetry groups [11, Lemma 3.7]. In this section, we study the groups of isometries in $\mathbb{E}^{3}$ of discrete chiral polyhedra as well as some groups of discrete regular polyhedra with infinite irreducible symmetry groups. Such groups of isometries must be crystallographic (that is, discrete groups of isometries having compact fundamental region) [10, Lemma 4.1].

For the purposes of this section we shall assume that $\mathcal{K}$ is a discrete chiral or discrete infinite pure regular polyhedron in $\mathbb{E}^{3}$. Let $G=G(\mathcal{K})$ be the subgroup of the symmetry group of $\mathcal{K}$ generated by rotations $S_{1}$ and $S_{2}$ specified in the previous section. Note that if $\mathcal{K}$ is orientably regular then $G$ is an index two subgroup in the symmetry group, otherwise $G$ is the whole symmetry group.

Let $\mathcal{T}(\mathcal{K})$ be the translation subgroup of $G$. It follows from [8, Sect. 7E], [10] and [11] that the subgroup has three linearly independent generators, and that the quotient of $G$ by $\mathcal{T}(\mathcal{K})$, called the special group of $G$, must be one of the following groups

$$
[3,4]^{+}, \quad[3,3]^{+}, \quad[3,4], \quad[3,3] \text { or }[3,3]^{*} \text {, }
$$

where $[3,3]^{*}=[3,3]^{+} \times\langle Z\rangle$ and $Z$ is the central inversion at the origin.

It is convenient to denote by $R^{\prime}$ the image of an element $R \in G$ under the quotient by $\mathcal{T}(\mathcal{K})$. Although strictly speaking $R^{\prime}$ is not an isometry, it will be useful to identify it with the corresponding isometry that fixes the origin. Note that in particular, $S_{1}^{\prime}$ and $S_{2}^{\prime}$ generate the special group of $G$. 
Following [8, pp. 166, 167] we denote by $\Lambda_{s}$ the sub-lattice of $\mathbb{Z}^{3}$ generated by $s$ and its images under permutations and changes of signs of its entries. As we shall see in the two subsequent sections, for some positive $d, \mathcal{T}(G)$ is

- $\Lambda_{(d, 0,0)}=d \mathbb{Z}^{3}$,

- $\Lambda_{(d, d, 0)}$, also called the face-centered cubic lattice, or

- $\Lambda_{(d, d, d)}$, also called the body-centered cubic lattice.

Given a subgroup $G$ of the symmetry group of $\mathcal{K}$ in terms of the isometries $S_{1}$ and $S_{2}$, we now develop a method, summarized in Theorem 3.2 below, of determining a set of defining relations for $G$.

The first step is to write the generating translations $t_{1}, t_{2}$ and $t_{3}$ of $\mathcal{T}(G)$ as words in $S_{1}$ and $S_{2}$. The next step is to find defining relations

$$
h_{i}\left(S_{1}^{\prime}, S_{2}^{\prime}\right)=\varepsilon, \quad \text { for } i=1, \ldots, k,
$$

for the special group of $G$ in terms of $S_{1}^{\prime}$ and $S_{2}^{\prime}$, where $h_{i}\left(S_{1}^{\prime}, S_{2}^{\prime}\right)$ stands for a fixed word in $S_{1}^{\prime}, S_{2}^{\prime}$. Note that whenever $h_{i}\left(S_{1}^{\prime}, S_{2}^{\prime}\right)=\varepsilon$, the word $h_{i}\left(S_{1}, S_{2}\right)$ corresponds to a translation. This translation is given by the word $f_{i}$ in Theorem 3.2.

In the proof of Theorem 3.2, we shall make use of the following well-known fact from group theory.

Lemma 3.1 Let $F$ be the free group on $k_{1}, \ldots, k_{m}$ and $K$ a finite group generated by $k_{1}, \ldots, k_{m}$ with a set of defining relations given by $r_{i}=\varepsilon$ for $i=1, \ldots, n$ for some words $r_{1}, \ldots, r_{n}$ in $\left\{k_{1}, \ldots, k_{m}\right\}$. Then, for any two words $w$ and $\hat{w}$ in $F$ representing the same element in $K, \hat{w}=w \beta$ for some $\beta$ in the normal closure of $\left\{r_{1}, \ldots, r_{n}\right\}$ in $F$.

The following theorem specifies a set of defining relations for $G=\left\langle S_{1}, S_{2}\right\rangle$ (some of which may be superfluous as we shall see in Sects. 4 and 5).

Theorem 3.2 Let $G=\left\langle S_{1}, S_{2}\right\rangle$ be the symmetry group of a discrete geometrically chiral polyhedron or the even subgroup of an infinite discrete pure regular polyhedron in the Euclidean 3-space. Let $\mathcal{T}(G)$ be its translation subgroup with generating set $\left\{t_{1}, t_{2}, t_{3}\right\}$ given in terms of $S_{1}$ and $S_{2}$, and let the defining relations of $G / \mathcal{T}(G)=$ $\left\langle S_{1}^{\prime}, S_{2}^{\prime}\right\rangle$ be given by (5).

Then the following constitute a set of defining relations for $G$

$$
\begin{aligned}
h_{i}\left(S_{1}, S_{2}\right) & =f_{i}, \quad \text { for } i=1, \ldots, k, \\
t_{i} t_{j} & =t_{j} t_{i}, \quad i, j=1,2,3, \\
S_{k}^{-1} t_{i} S_{k} & =f_{i, k}, \quad \text { for } i=1,2,3 \text { and } k=1,2,
\end{aligned}
$$

where $f_{i}$ and $f_{i, k}$ are fixed words in the generators of $\mathcal{T}(G)$.

Proof Let $G_{0}=\left\{g_{1}, \ldots, g_{m}\right\}$ be a set of representatives, one from each coset in $G / \mathcal{T}(G)$, and let $W:=\left\{w_{1}, \ldots, w_{m}\right\}$ be a set of words on the set $\left\{S_{1}, S_{2}\right\}$ such that $w_{s}$ represents the element $g_{s}$ for every $s \in\{1, \ldots, m\}$. Then every element in $G$ can be written in a unique form as $g_{i} t_{1}^{a} t_{2}^{b} t_{3}^{c}$ with $g_{i} \in G_{0}$ and $a, b, c \in \mathbb{Z}$. 
A set of relations is a set of defining relations for $G$ whenever these relations are sufficient to determine whether two arbitrary words in $S_{1}$ and $S_{2}$ represent the same element of $G$. Therefore, to show that (6), (7) and (8) constitute a set of defining relations for $G$, we only need to show that an arbitrary element $g_{w}$ of $G$ represented by a word $w$ in $S_{1}$ and $S_{2}$ can be written as $g_{i} t_{1}^{a} t_{2}^{b} t_{3}^{c}$ for some $g_{i} \in G_{0}$ and $a, b, c \in \mathbb{Z}$ using only these relations.

Following Lemma 3.1, with $K=G / \mathcal{T}(G)$, we note that the word $w$ in $S_{1}$ and $S_{2}$ can be written as $w=w_{i} \beta$ for some $i \in\{1, \ldots, m\}$ and $\beta$ in the normal closure of $\left\{h_{1}\left(S_{1}, S_{2}\right), \ldots, h_{k}\left(S_{1}, S_{2}\right)\right\}$ in the free group generated by $S_{1}$ and $S_{2}$. Assume $\beta=\left(z_{1}^{-1} h_{i_{1}}\left(S_{1}, S_{2}\right) z_{1}\right) \cdots\left(z_{n}^{-1} h_{i_{n}}\left(S_{1}, S_{2}\right) z_{n}\right)$ for some positive integer $n$ and some $z_{1}, \ldots, z_{n}$ in the free group. Using (6), it follows that $g_{w}=$ $g_{i}\left(z_{1}^{-1} f_{i_{1}} z_{1}\right) \cdots\left(z_{n}^{-1} f_{i_{n}} z_{n}\right)$ for some $i_{1}, \ldots, i_{n} \in\{1, \ldots, k\}$. Now (8) implies $t_{i} S_{k}=$ $S_{k} f_{i, k}$ where $f_{i, k} \in \mathcal{T}(G)$. Since each $f_{i_{j}} \in \mathcal{T}(G)$, we can repeatedly use (8) to show that $z_{j}^{-1} f_{i_{j}} z_{j}=z_{j}^{-1} z_{j} \hat{f}_{i_{j}}=\hat{f}_{i_{j}}$ for some $\hat{f}_{i_{j}} \in \mathcal{T}(G)$ implying that $g_{w}=$ $g_{i} \hat{f}_{i_{1}} \cdots \hat{f}_{i_{n}}=g_{i} t$ for some $t \in \mathcal{T}(G)$. Note that to express each $\hat{f}_{i_{j}}$ and $t$ in the form $t_{1}^{a} t_{2}^{b} t_{3}^{c}$ we need to make use of relations (7) and of the fact that $f_{i}$ and $f_{j, k}$ are words in $t_{1}, t_{2}, t_{3}$.

Note that this theorem can be stated for more general groups with a finite index normal subgroup but we shall only need it in this restricted form.

We conclude the section with two elementary lemmas which we will require in the following sections.

Lemma 3.3 Let $R$ be the rotation in $\mathbb{E}^{3}$ given by

$$
(x, y, z) R=(y, z, x) \text {, }
$$

and let $T$ be a translation by a vector $(a, b, c)$. Then $R^{-1} T R$ and $R T R^{-1}$ are the translations by the vectors $(b, c, a)$ and $(c, a, b)$, respectively.

Lemma 3.4 Let $S$ be the rotatory reflection in $\mathbb{E}^{3}$ given by

$$
(x, y, z) S=(-z,-x,-y),
$$

let $T_{1}$ be the translation by the vector $(a, b, c)$, and let $T_{2}$ and $T_{3}$ be the translations by the vectors $(c, a, b)$ and $(b, c, a)$, respectively (that is, $T_{2}=R T R^{-1}$ and $T_{3}=$ $R^{-1} T R$ with $R$ as in Lemma 3.3). Then

$$
\begin{aligned}
& S^{-1} T_{1} S=T_{2}^{-1}, \\
& S^{-1} T_{2} S=T_{3}^{-1}, \\
& S^{-1} T_{3} S=T_{1}^{-1} .
\end{aligned}
$$

Proof This follows directly from the properties

- $S^{-1} T_{i} S=Z T_{i+1} Z$ (with the index taken modulo 3), and

- if $T$ is the translation by the vector $v$ then $Z T Z=-T$. 


\section{The Polyhedra with Finite Faces}

There are three families of discrete chiral polyhedra in Euclidean 3-space with finite faces. One family consists of the self-dual polyhedra of Schläfli type $\{6,6\}$. The second family consist of the polyhedra with Schläfli type $\{4,6\}$, while the third one contains all their duals. We follow Schulte in [10], extending these families in such a way that certain regular polyhedra are included, and denoting the families by $P(a, b)$, $Q(c, d)$ and $Q_{1}(c, d)$, respectively. In this section, we determine when these polyhedra are combinatorially chiral and in addition we give presentations for their automorphism groups.

\subsection{The Type $\{6,6\}$}

Consider the group $G(a, b)$ of isometries in $\mathbb{E}^{3}$ generated by $S_{1}$ and $S_{2}=S_{2}^{\prime}$ given by

$$
\begin{aligned}
& (x, y, z) S_{1}=(-y, z-b, x-a), \\
& (x, y, z) S_{2}=(-z,-x,-y),
\end{aligned}
$$

(see [10, Sect. 5]). Let $Z=-\varepsilon$ be the reflection in the origin $o$ of $\mathbb{E}^{3}$. Then $S_{2}^{3}=Z$ and $S_{2}^{2}$ is the rotation $R$ in Lemma 3.3.

Since $\left(S_{1} S_{2}\right)^{2}=\varepsilon$ and $\left\langle S_{1}\right\rangle \cap\left\langle S_{2}\right\rangle=\{\varepsilon\}$ then, as explained in Sect. 2, one can use the group $G(a, b)$ to construct an abstract orientably regular or chiral polytope $P(a, b)$. Wythoff's construction (see [8, Theorem 5A10] and [10, Sect. 2]) on $G(a, b)$ with the origin $o$ as initial vertex can then be used to obtain the geometric polyhedron $P(a, b)$, which may be orientably regular or chiral. Note that $G(a, b)$ is the symmetry group of $P(a, b)$ if and only if $P(a, b)$ is geometrically chiral.

In [10], it is proved that whenever $a \neq \pm b$, the polyhedron $P(a, b)$ is geometrically chiral and has skew hexagons as faces and vertex-figures. If $a= \pm b$ the polyhedron $P(a, b)$ is geometrically regular (that is, $S_{1}$ and $S_{2}$ generate the even subgroup of its symmetry group). The polyhedron $P(1,1)$ is isomorphic to the Petrie-Coxeter regular polyhedron $\{6,6 \mid 3\}$ with planar faces and skew vertex-figures. On the other hand, $P(1,-1)$ is isomorphic to Grünbaum's regular polyhedron $\{6,6\}_{4}$ with skew faces and planar vertex-figures.

The special group of the chiral polyhedra $P(a, b)$ is isomorphic to $[3,3]^{*}$. Let $\rho_{0}, \rho_{1}, \rho_{2}$ be the distinguished generators of $[3,3]$. It follows that

$$
[3,3]^{*}=\left\langle S_{1}^{\prime}, S_{2} \mid\left(S_{1}^{\prime}\right)^{6}=S_{2}^{6}=\left(S_{1}^{\prime} S_{2}\right)^{2}=\left(S_{1}^{\prime}\right)^{3} S_{2}^{3}=\varepsilon\right\rangle,
$$

is a presentation for $[3,3]^{*}$, where $S_{1}^{\prime}=\rho_{0} \rho_{1} Z$ and $S_{2}=\rho_{1} \rho_{2} Z$. Note that $S_{1}^{\prime}$ and $S_{2}$ are rotatory reflections with a rotation angle of $\pi / 3$.

Schulte proved that the polyhedron $P(a, b)$ is discrete if and only if $a$ or $b$ is zero or $a$ is a rational multiple of $b$ ([10, Lemma 5.2]). Moreover, it follows from the definitions of $S_{1}$ and $S_{2}$ that the polyhedron $P(a, b)$ is similar (and thus, combinatorially equal) to the polyhedron $P(s a, s b)$ for any non-zero real number $s$. Therefore, we may assume that $a, b$ are relatively prime integers. Furthermore, Shulte 
proved that the polyhedron $P(a, b)$ is combinatorially isomorphic to the polyhedron $P(c, d)$ if and only if $(a, b)=s(c, d)$ or $(a, b)=s(d, c)$ for some non-zero real $s$ (see [10, Theorem 5.18]). It follows that without loss of generality, we may assume that $a$ is an odd integer.

Let $\mathcal{T}(P(a, b))$ denote the translation subgroup of the symmetry group of $P(a, b)$. The translations by the vectors $(4 a, 0,0)$ and $(-4 b, 0,0)$ can be, respectively, written as

$$
\begin{aligned}
\tau_{a} & =\left(S_{2}^{-2} S_{1}^{2} S_{2}^{3} S_{1}^{-1}\right)^{2}, \\
\tau_{b} & =S_{1}^{2} S_{2}^{-2} S_{1} S_{2}^{-1} S_{1} S_{2}^{3} S_{1} S_{2}^{3} S_{1}^{-1} S_{2} .
\end{aligned}
$$

The following theorem implies that, up to similarity, there are only two regular polyhedra in the family of polyhedra $P(a, b)$, namely $P(1,1)$ and $P(1,-1)$. Note that, as abstract polyhedron $P(1,-1)$ is self-dual since the geometric polyhedron $P(1,-1)$ is similar to $P(-1,1)$.

Theorem 4.1 The polyhedron $P(a, b)$ is combinatorially regular if and only if $a= \pm b$.

Proof In [10, Theorem 5.16], Schulte proves that, for $a= \pm b$, the polyhedra $P(a, b)$ are geometrically regular (and thus, combinatorially regular). We proceed to prove that these are the only two cases where $P(a, b)$ is a regular abstract polyhedron.

An easy calculation will show that the enantiomorphic words $\tau_{a}^{*}=\left(S_{2}^{2} S_{1}^{-2} S_{2}^{3} S_{1}\right)^{2}$ and $\tau_{b}^{*}=S_{1}^{-2} S_{2}^{2} S_{1}^{-1} S_{2} S_{1}^{-1} S_{2}^{3} S_{1}^{-1} S_{2}^{3} S_{1} S_{2}^{-1}$ of $\tau_{a}$ and $\tau_{b}$ as defined above, correspond to the translations $S_{2}^{2} \tau_{b} S_{2}^{-2}$ and $S_{2}^{2} \tau_{a} S_{2}^{-2}$ by vectors $(0,-4 b, 0)$ and $(0,4 a, 0)$, respectively.

If the polyhedron $P(a, b)$ is combinatorially regular, then the enantiomorphic element $\tau_{a b}^{*}$ of the translation $\tau_{a b}=\tau_{a}^{b}=\tau_{b}^{-a}$ by the vector $(4 a b, 0,0)$ must satisfy

$$
\tau_{a b}^{*}=\left(S_{2}^{2} \tau_{b} S_{2}^{-2}\right)^{b}=\left(S_{2}^{2} \tau_{a} S_{2}^{-2}\right)^{-a} .
$$

However, $\left(S_{2}^{2} \tau_{b} S_{2}^{-2}\right)^{b}$ corresponds to the translation by the vector $\left(0,-4 b^{2}, 0\right)$, and $\left(S_{2}^{2} \tau_{a} S_{2}^{-2}\right)^{-a}$ to the translation by the vector $\left(0,-4 a^{2}, 0\right)$, implying that $a= \pm b$.

To derive the defining relations for the polyhedron $P(a, b)$ we first determine a generating set $\left\{t_{1}, t_{2}, t_{3}\right\}$ of the translation group of $P(a, b)$ and then make use of Theorem 3.2. The group $\mathcal{T}(P(a, b))$ is one of the following

(a) $\Lambda_{(1,1,1)}$ if $b$ is even,

(b) $2 \mathbb{Z}^{3}$ if $a+b \equiv 0(\bmod 4)$, or

(c) $2 \Lambda_{(1,1,0)}$ if $a \equiv b(\bmod 4)$

(see [10, Lemma 5.5]).

We define the translation $t_{1}$ in (a), (b) and (c) to be the translation by the vector $(-1,1,1),(2,0,0)$ and $(2,2,0)$, respectively. We then let $t_{2}=S_{2}^{2} t_{1} S_{2}^{-2}$ and $t_{3}=$ $S_{2}^{-2} t_{1} S_{2}^{2}$ (see Lemma 3.3 and Table 1). A simple calculation shows that $\mathcal{T}(P(a, b))=$ $\left\langle t_{1}, t_{2}, t_{3}\right\rangle$. 
Table 1 The generators of $\mathcal{T}(P(a, b))$

\begin{tabular}{llll}
\hline Translation & $b$ even & $a+b \equiv 0(\bmod 4)$ & $a \equiv b(\bmod 4)$ \\
\hline$t_{1}$ & $(-1,1,1)$ & $(2,0,0)$ & $(2,2,0)$ \\
$t_{2}$ & $(1,-1,1)$ & $(0,2,0)$ & $(0,2,2)$ \\
$t_{3}$ & $(1,1,-1)$ & $(0,0,2)$ & $(2,0,2)$ \\
\hline
\end{tabular}

It is convenient to make use of the translations

$$
\begin{aligned}
& \alpha:=\left(S_{1} S_{2}^{-1}\right)^{3}, \\
& \beta:=\left(S_{2}^{-2} S_{1}^{2}\right)^{2} S_{2} S_{1}^{2} S_{2}^{3},
\end{aligned}
$$

by the vectors $(-a+b, a-b, a-b),(2 a, 0,-2 b)$, respectively. Denoting by $\tau_{1}, \tau_{2}$ and $\tau_{3}$ the translations by the vectors $(4,0,0),(0,4,0)$ and $(0,0,4)$, respectively, and using Lemma 3.3, we have that

$$
\begin{aligned}
& \tau_{1}=\tau_{a}^{\lambda} \tau_{b}^{-\mu} \quad \text { with } \lambda a+\mu b=1, \\
& \tau_{2}=S_{2}^{2} \tau_{1} S_{2}^{-2}, \\
& \tau_{3}=S_{2}^{-2} \tau_{1} S_{2}^{2} .
\end{aligned}
$$

In order to determine the defining relations for the symmetry group of $P(a, b)$ in terms of the generators $S_{1}$ and $S_{2}$, we need to express each $t_{i}, i=1,2,3$ in terms of $S_{1}$ and $S_{2}$. We do this in the three cases below where we make use of the elements $\alpha, \beta, \tau_{1}, \tau_{2}, \tau_{3}$ given above in terms of $S_{1}$ and $S_{2}$. Furthermore, in the first case we define $\gamma$ in terms of $\beta, \tau_{1}$ and $\tau_{3}$, and hence also in terms of $S_{1}$ and $S_{2}$.

In case $b$ is even, say $b=2 l$, and assuming that $a=2 k+1$ for some $k$, the automorphism $\gamma:=\beta \tau_{1}^{-k} \tau_{3}^{l}$ corresponds to the translation by the vector $(2,0,0)$. Then $\alpha \gamma^{-k+l} S_{2}^{2} \gamma^{k-l} S_{2}^{-2} S_{2}^{-2} \gamma^{k-l} S_{2}^{2}$ is the translation by the vector $(-1,1,1)$, implying that

$$
t_{1}=\alpha \gamma^{-k+l} S_{2}^{2}\left(\gamma^{k-l} S_{2}^{2}\right)^{2} .
$$

In case $a+b \equiv 0(\bmod 4)$, say $a=4 k+1$ and $b=4 l+3$ for some integers $k$ and $l$, the required translation by the vector $(2,0,0)$ can be found to be

$$
t_{1}=S_{2}^{-2} \beta \alpha \beta \tau_{1}^{k+l+1} \tau_{2}^{l-k+1} \tau_{3}^{3 l-k+2} S_{2}^{2} .
$$

Finally, if $a=4 k+c, b=4 l+c$ for some integers $k, l$ and for some $c \in\{1,3\}$, the translation by $(2,2,0)$ can be expressed as

$$
t_{1}=S_{2}^{2} \beta \tau_{1}^{-2 k+(1-c) / 2} \tau_{3}^{2 l+(c+1) / 2} S_{2}^{-2} .
$$

The following theorem gives a set of defining relations for the chiral polyhedra $P(a, b)$.

Theorem 4.2 Let $P(a, b)$ be a geometrically chiral polyhedron where $a$ and $b$ are relatively prime integers with a odd. Let $t_{1}$ be the translation given in (10), (11) 
Table 2 Words on the generating translations for $P(a, b)$

\begin{tabular}{llll}
\hline Word & $b$ even & $a+b \equiv 0(\bmod 4)$ & $a \equiv b(\bmod 4)$ \\
\hline$w_{1}$ & $t_{2}^{-1}$ & $t_{3}$ & $t_{1}^{-1} t_{2}$ \\
$w_{2}$ & $t_{1} t_{2} t_{3}$ & $t_{1}^{-1}$ & $t_{2} t_{3}^{-1}$ \\
$w_{3}$ & $t_{1}^{a} t_{2}^{-b}$ & $t_{1}^{-(a+b) / 2} t_{2}^{(a+b) / 2} t_{3}^{(a-b) / 2}$ & $t_{1}^{(b-a) / 4} t_{2}^{(3 a+b) / 4} t_{3}^{(-a-3 b) / 4}$ \\
\hline
\end{tabular}

or (12), $t_{2}=S_{2}^{2} t_{1} S_{2}^{-2}$ and $t_{3}=S_{2}^{-2} t_{1} S_{2}^{2}$, each expressed in terms of $S_{1}, S_{2}$. A set of defining relations for the symmetry group $G(a, b)=\left\langle S_{1}, S_{2}\right\rangle$ of $P(a, b)$ is given by $S_{1}^{6}=S_{2}^{6}=\left(S_{1} S_{2}\right)^{2}=\varepsilon$ together with

$$
\begin{aligned}
t_{1} t_{2} & =t_{2} t_{1}, \\
S_{2}^{3} t_{1} S_{2}^{3} & =t_{1}^{-1}, \\
S_{1}^{-1} t_{1} S_{1} & =w_{1}\left(t_{1}, t_{2}, t_{3}\right), \\
S_{1}^{-1} t_{2} S_{1} & =w_{2}\left(t_{1}, t_{2}, t_{3}\right), \\
S_{1}^{3} S_{2}^{3} & =w_{3}\left(t_{1}, t_{2}, t_{3}\right),
\end{aligned}
$$

where $w_{1}, w_{2}, w_{3}$ are the words on $t_{1}, t_{2}, t_{3}$ given in Table 2.

Proof According to Theorem 3.2, the defining relations for the symmetry group of $P(a, b)$, in addition to the relations derived from those of the special group $[3,3]^{*}$, are given in (7) and (8). The only defining relation in (9) we need to consider is $S_{1}^{3} S_{2}^{3}=\varepsilon$. The relation (17), with $w_{3}$ given in Table 2 for each of the choices of $a$ and $b$, can easily be verified (geometrically) using the generating translations in Table 1.

The commutativity of $t_{3}$ with $t_{1}$ and $t_{2}$ can be obtained by conjugating (13) by $S_{2}^{-2}$ and $S_{2}^{2}$ respectively, implying that any two elements in $\left\langle t_{1}, t_{2}, t_{3}\right\rangle$ commute.

The definition of $t_{1}, t_{2}$ and $t_{3}$ together with Lemma 3.4 imply that, as symmetries of $P(a, b)$, these translations and the rotatory reflection $S_{2}$ must satisfy the following relations

$$
S_{2}^{-1} t_{i} S_{2}=t_{i+1}^{-1},
$$

with the subscripts taken modulo 3 . We note that these relations are implied by (14), which is equivalent to $S_{2} t_{2} S_{2}^{-1}=t_{1}^{-1}$, whereas the other two are obtained by conjugating this relation by $S_{2}^{2}$ and $S_{2}^{-2}$.

The expressions for the conjugation of $t_{1}$ and $t_{2}$ by $S_{1}$ given in relations (15) and (16) can also be verified geometrically.

To show that the relation determined by the conjugation of $t_{3}$ by $S_{1}$ is redundant we first observe that relation (17) implies that

$$
S_{1}^{3}=w_{3}\left(t_{1}, t_{2}, t_{3}\right) S_{2}^{3}=S_{2}^{3} w_{3}\left(t_{1}, t_{2}, t_{3}\right)^{-1} \text {. }
$$

This in turn implies $S_{1}^{3} t S_{1}^{3}=S_{2}^{3} t S_{2}^{3}=t^{-1}$ for any $t \in\left\langle t_{1}, t_{2}, t_{3}\right\rangle$. For $b$ even, 


$$
t_{1}^{-1}=S_{1}^{3} t_{1} S_{1}^{3}=S_{1}^{-2}\left(t_{2}^{-1}\right) S_{1}^{2}=S_{1}^{-1}\left(t_{1} t_{2} t_{3}\right)^{-1} S_{1}=t_{2}\left(t_{1}^{-1} t_{2}^{-1} t_{3}^{-1}\right) S_{1}^{-1} t_{3}^{-1} S_{1},
$$

implying that $S_{1}^{-1} t_{3} S_{1}=t_{3}^{-1}$. Similarly, $S_{1}^{-1} t_{3} S_{1}=t_{2}$ can be derived for $b$ odd.

We note that some of the relations in Theorem 4.2 may be superfluous. For example, for $(a, b)=(1,0)$, we observe that $t_{1}=S_{1}^{3} S_{2}^{3}$ and hence that the relation (17) is not needed. Moreover, relation (14) is trivial and relations (15) and (16) are equivalent. It is not difficult to see that (15) implies (13), and hence the only defining relation for $P(1,0)$ in addition to those relations given by the Schläfli type, is $(15)$, which is equivalent to

$$
S_{1} S_{2}^{2} S_{1}^{-1} S_{2} S_{1}^{2} S_{2}^{-1}=\varepsilon .
$$

From this relation one can verify directly that the polyhedron $P(1,0)$ is chiral by verifying that the enantiomorphic word of the word on the left of the equation above is not the identity.

\subsection{The Types $\{4,6\}$ and $\{6,4\}$}

The family of discrete polyhedra in $\mathbb{E}^{3}$ with Schläfli type $\{6,4\}$ consists of the dual of the discrete polyhedra with Schläfli type $\{4,6\}$. We need only consider the latter since one can derive the defining relations for the symmetry group of the dual by replacing each $\sigma_{1}$ by $\sigma_{2}^{-1}$ and $\sigma_{2}$ by $\sigma_{1}^{-1}[11$, Sect. 3].

We now construct the geometric polyhedron $Q(c, d)$ from the group of isometries $G(c, d)$ generated by

$$
\begin{aligned}
& (x, y, z) S_{1}=(-x+c, z-d,-y-c), \\
& (x, y, z) S_{2}=(-z,-x,-y),
\end{aligned}
$$

following [10] and the same argument as in the previous subsection.

Note that $S_{2}=S_{2}^{\prime}$ is the same element for the polyhedron $Q(c, d)$ and the polyhedron $P(a, b)$, and thus $S_{2}^{3}=Z$ and $S_{2}^{2}$ is the rotation $R$ in Lemma 3.3. It was proved in [10] that whenever $c d \neq 0$, the polyhedron $Q(c, d)$ is geometrically chiral and has skew 4-gons as faces and skew hexagons as vertex-figures. If $c d=0$ the polyhedron $Q(c, d)$ is geometrically regular and $S_{1}$ and $S_{2}$ generate the even subgroup of its symmetry group. The polyhedron $Q(1,0)$ is isomorphic to the Petrie-Coxeter regular polyhedron $\{4,6 \mid 4\}$ with planar faces and skew vertex-figures. On the other hand, $Q(0,1)$ is isomorphic to Grünbaum's regular polyhedron $\{4,6\}_{6}$ with skew faces and planar vertex-figures.

The special group of $Q(c, d)$ is isomorphic to [3,4]. Note that $S_{1}^{\prime}$ and $S_{2}$ are rotatory reflections in $[3,4]$ of orders 4 and 6 such that $\left(S_{1}^{\prime} S_{2}\right)^{2}=\varepsilon$ (see $[10$, Lemma 6.1]). It follows that

$$
[3,4]=\left\langle S_{1}^{\prime}, S_{2} \mid\left(S_{1}^{\prime}\right)^{4}=S_{2}^{6}=\left(S_{1}^{\prime} S_{2}\right)^{2}=\left(S_{1}^{\prime} S_{2}^{-2}\right)^{2}=\varepsilon\right\rangle
$$

is a presentation for the special group of $Q(c, d)$.

The polyhedron $Q(c, d)$ is discrete if and only if $c$ or $d$ is zero or $c$ is a rational multiple of $d$ ([10, Lemma 6.2]). It follows from the definitions of $S_{1}$ and $S_{2}$ that the 
polyhedron $Q(c, d)$ is similar (and thus, combinatorially equal) to the polyhedron $Q(s c, s d)$ for any non-zero real number $s$. Therefore, without loss of generality, we may assume that $c, d$ are relatively prime integers. Furthermore, Schulte proved that the polyhedron $Q(c, d)$ is combinatorially isomorphic to the polyhedron $Q(e, f)$ if and only if $(c, d)=s(e, f)$ or $(c, d)=s(-e, f)$ for some non-zero real $s$ (see [10, Theorem 6.13]).

Let $\mathcal{T}(Q(c, d))$ be the translation subgroup of the symmetry group of $Q(c, d)$. The translations by the vectors $(4 c, 0,0),(0,4 c, 0),(0,0,4 c),(-2 d, 2 d,-2 d)$ can be respectively written as

$$
\begin{aligned}
& \kappa_{1}:=S_{2}^{-2}\left(S_{1} S_{2}^{-1}\right)^{4} S_{2}^{2}, \\
& \kappa_{2}:=\left(S_{1} S_{2}^{-1}\right)^{4}, \\
& \kappa_{3}:=\left(S_{1}^{-1} S_{2}\right)^{4}, \\
& \kappa_{4}:=\left(S_{1}^{2} S_{2}^{2}\right)^{3} .
\end{aligned}
$$

The theorem below implies that, up to similarity, there are only two regular polyhedra in the family of polyhedra $Q(c, d)$, namely $Q(0,1)$ and $Q(1,0)$.

Theorem 4.3 The polyhedron $Q(c, d)$ is combinatorially regular if and only if $c d=0$.

Proof The polyhedron $Q(c, d)$ is geometrically regular (and thus combinatorially regular) if $c d=0$ (see [10, Theorem 6.11]).

Conversely, assuming that $Q(c, d)$ is regular, the enantiomorphic elements of $\kappa_{1}, \kappa_{2}, \kappa_{3}, \kappa_{4}$ are respectively $\kappa_{1}, \kappa_{3}, \kappa_{2}$, and the translation by the vector $(2 d$, $2 d,-2 d)$.

It is straightforward to check that the translation $\kappa_{c d}$ by the vector $(4 d c, 0,0)$ can be written as

$$
\kappa_{1}^{d}=\kappa_{2}^{d} \kappa_{3}^{-d} \kappa_{4}^{-2 c} .
$$

Since $Q(c, d)$ is regular, we can write the enantiomorphic element of $\kappa_{c d}$ as $\left(\kappa_{1}^{*}\right)^{d}=$ $\left(\kappa_{2}^{*}\right)^{d}\left(\kappa_{3}^{*}\right)^{-d}\left(\kappa_{4}^{*}\right)^{-2 c}$. But $\kappa_{1}^{d}=\left(\kappa_{1}^{*}\right)^{d}$ is the translation by the vector $(4 c d, 0,0)$ while $\left(\kappa_{2}^{*}\right)^{d}\left(\kappa_{3}^{*}\right)^{-d}\left(\kappa_{4}^{*}\right)^{-2 c}$ can easily be seen to be the translation by the vector $(-4 d c,-8 c d, 8 c d)$. Thus $c d=0$.

To derive the defining relations for the polyhedra $Q(c, d)$ we determine a generating set $\left\{t_{1}, t_{2}, t_{3}\right\}$ of the translation subgroup $\mathcal{T}(Q(c, d))$ of $Q(c, d)$ and then make use of Theorem 3.2. The group $\mathcal{T}(Q(c, d))$ is one of the following

(a) $2 \Lambda_{(1,1,0)}$ if $d$ is even,

(b) $2 \mathbb{Z}^{3}$ if $c$ is even, or

(c) $2 \Lambda_{(1,1,1)}$ if $c$ and $d$ are odd

(see [10, Lemma 6.5]).

We choose the vectors $(2,2,0),(2,0,0)$ and $(-2,2,2)$ for $t_{1}$ in $(a),(b)$ and $(c)$, respectively, and let $t_{2}=S_{2}^{2} t_{1} S_{2}^{-2}$ and $t_{3}=S_{2}^{-2} t_{1} S_{2}^{2}$ (see Lemma 3.3 and Table 3). 
Table 3 The generators of $\mathcal{T}(Q(c, d))$

\begin{tabular}{llll}
\hline Translation & $d$ even & $c$ even & $c$ and $d$ odd \\
\hline$t_{1}$ & $(2,2,0)$ & $(2,0,0)$ & $(-2,2,2)$ \\
$t_{2}$ & $(0,2,2)$ & $(0,2,0)$ & $(2,-2,2)$ \\
$t_{3}$ & $(2,0,2)$ & $(0,0,2)$ & $(2,2,-2)$ \\
\hline
\end{tabular}

Essentially the same calculation as above for $\mathcal{T}(P(a, b))$ carries over to show that $\mathcal{T}(Q(c, d))=\left\langle t_{1}, t_{2}, t_{3}\right\rangle$.

Assuming that $\lambda$ and $\mu$ are such that $\lambda c+\mu d=1$, we introduce the translations

$$
\begin{aligned}
& \vartheta:=\left(S_{2}^{2} S_{1}^{-1}\right)^{2}, \\
& \xi_{1}:=S_{2}^{2} \kappa_{3}^{\lambda}\left(\vartheta^{2} \kappa_{1}^{-1} \kappa_{2}\right)^{\mu} S_{2}^{-2}, \\
& \xi_{2}:=S_{2}^{-2} \kappa_{3}^{\lambda}\left(\vartheta^{2} \kappa_{1}^{-1} \kappa_{2}\right)^{\mu} S_{2}^{2}, \\
& \xi_{3}:=\kappa_{3}^{\lambda}\left(\vartheta^{2} \kappa_{1}^{-1} \kappa_{2}\right)^{\mu},
\end{aligned}
$$

by vectors $(2 c,-2 c, 2 d),(4,0,0),(0,4,0)$ and $(0,0,4)$, respectively.

To determine the defining relations for the polyhedron $Q(c, d)$, we use the same idea as above for $P(a, b)$ and express $t_{1}$ in terms of $S_{1}$ and $S_{2}$. This time we make use of the translations $\vartheta, \xi_{1}, \xi_{2}$ and $\xi_{3}$, expressed above in terms of $S_{1}$ and $S_{2}$.

Assuming $d$ is even, say $d=2 l$, and $c$ is odd, say $c=2 k+1$ for some integers $l, k$, we can verify that the required translation by the vector $(2,2,0)$ can be written as

$$
t_{1}=\vartheta \xi_{1}^{-k} \xi_{2}^{k+1} \xi_{3}^{-l}
$$

Similarly, if $c$ is even, say $c=2 k$, and assuming that $d=2 l+1$ for some integers $k, l$, we note that the translation by the vector $(2,0,0)$ can be found to be

$$
t_{1}=S_{2}^{2} \vartheta \xi_{1}^{-k} \xi_{2}^{k} \xi_{3}^{-l} S_{2}^{-2}
$$

Finally, if both $c$ and $d$ are odd, say $c=2 k+1$ and $d=2 l+1$, for some integers $k, l$, then the translation by the vector $(-2,2,2)$ can be expressed as

$$
t_{1}=\vartheta \xi_{1}^{-k-1} \xi_{2}^{k+1} \xi_{3}^{-l}
$$

The following theorem gives a set of defining relations for the chiral polyhedron $Q(c, d)$. As in the case of the polyhedra $P(a, b)$, some of the relations given in the theorem may be superfluous.

Theorem 4.4 Let $Q(c, d)$ be a geometrically chiral polyhedron where $c$ and $d$ are relatively prime integers. Let $t_{1}$ be the translation given in (18), (19) or (20), $t_{2}=$ $S_{2}^{2} t_{1} S_{2}^{-2}$ and $t_{3}=S_{2}^{-2} t_{1} S_{2}^{2}$, each expressed in terms of $S_{1}$ and $S_{2}$. A set of defining relations for the symmetry group $G(c, d)=\left\langle S_{1}, S_{2}\right\rangle$ of $Q(c, d)$ is given by $S_{1}^{4}=S_{2}^{6}=$ $\left(S_{1} S_{2}\right)^{2}=\varepsilon$ together with 
Table 4 Words on the generating translations for $Q(c, d)$

\begin{tabular}{llll}
\hline Word & $d$ even & $c$ even & $c$ and $d$ odd \\
\hline$w_{1}$ & $t_{1}^{d / 2} t_{2}^{c-d / 2} t_{3}^{-c-d / 2}$ & $t_{1}^{-c} t_{2}^{c} t_{3}^{-d}$ & $t_{1}^{(c-d) / 2} t_{2}^{(-c-d) / 2}$ \\
$w_{2}$ & $t_{1} t_{3}^{-1}$ & $t_{3}^{-1}$ & $t_{1}$ \\
$w_{3}$ & $t_{2} t_{3}^{-1}$ & $t_{2}$ & $t_{1}$ \\
\hline
\end{tabular}

$$
\begin{aligned}
t_{1} t_{2} & =t_{2} t_{1}, \\
S_{2}^{3} t_{1} S_{2}^{3} & =t_{1}^{-1}, \\
S_{1}^{-1} t_{2} S_{1} & =w_{2}\left(t_{1}, t_{2}, t_{3}\right), \\
S_{1}^{-1} t_{3} S_{1} & =w_{3}\left(t_{1}, t_{2}, t_{3}\right), \\
\left(S_{1} S_{2}^{-2}\right)^{2} & =w_{1}\left(t_{1}, t_{2}, t_{3}\right),
\end{aligned}
$$

where $w_{2}, w_{3}, w_{4}$ are the words on $t_{1}, t_{2}, t_{3}$ given in Table 4.

Proof The proof is essentially the same as that of Theorem 4.2. The words $w_{1}, w_{2}$ and $w_{3}$ can be verified (geometrically) using the generating translations in Table 3. We only need to prove that the relation determined by the conjugation of $t_{1}$ by $S_{1}$ is redundant. To do this we observe that (21) and (25) imply that

$$
S_{1}^{-1} S_{2}^{2} S_{1}^{-1} t_{i} S_{1} S_{2}^{-2} S_{1}=S_{2}^{-2} t_{i} S_{2}^{2}=t_{i-1}
$$

for $i=1,2,3$ (with the subscripts taken modulo 3). In particular, for $d$ even we have that

$$
\begin{aligned}
t_{1} & =S_{1}^{-1} S_{2}^{2} S_{1}^{-1} t_{2} S_{1} S_{2}^{-2} S_{1}=S_{1}^{-1} S_{2}^{2} t_{1} t_{3}^{-1} S_{2}^{-2} S_{1} \\
& =S_{1}^{-1} t_{1}^{-1} t_{2} S_{1}=S_{1}^{-1} t_{1}^{-1} S_{1} S_{1}^{-1} t_{2} S_{1}=S_{1}^{-1} t_{1}^{-1} S_{1} t_{1} t_{3}^{-1},
\end{aligned}
$$

implying that $S_{1}^{-1} t_{1} S_{1}=t_{3}^{-1}$. Similarly, when $c$ is even or when both $c$ and $d$ are odd, we can derive $S_{1}^{-1} t_{1} S_{1}=t_{1}^{-1}$ and $S_{1}^{-1} t_{1} S_{1}=t_{3}$, respectively.

In conclusion, let us note that a simple calculation will show that a set of defining relations for the symmetry group of $Q(1,1)$ is given by the addition of

$$
S_{2}^{2} S_{1}^{-1} S_{2}^{3} S_{1} S_{2}^{-1} S_{1} S_{2}^{-2} S_{1}=\varepsilon
$$

to those relations implied by its Schläfli type.

\section{The Polyhedra with Infinite Faces}

There are three families of discrete chiral polyhedra in Euclidean 3-space with infinite faces (see [11]). Two of the families consist of polyhedra with Schläfli type 
$\{\infty, 3\}$. These families are geometrically and, as we shall see, combinatorially distinct. The third family consists of polyhedra with Schläfli type $\{\infty, 4\}$. Once again it will be convenient to extend the families in such a way that certain regular polyhedra are included in the families. In [11], the polyhedra in these families are denoted by $P_{i}(u, v)$, where $i=1,2,3$ denotes the family and $u$ and $v$ are parameters.

As in the cases of discrete chiral polyhedra in Euclidean 3-space with finite faces, the polyhedron $P_{i}(u, v)$ is geometrically similar to $P_{i}(s u, s v)$ for any non-zero real number $s$. However, the parameters $u$ and $v$ need not be rational multiples of each other, as was the case in the families of polyhedra with finite faces. This will allow us to continuously transform any chiral polyhedron in a family into any other chiral polyhedron in the same family.

\subsection{Type $\{\infty, 3\}$ over Squares}

We begin by considering the family consisting of polyhedra $P_{2}(c, d)$ built using Wythoff's construction from the group generated by

$$
\begin{aligned}
& (x, y, z) S_{1}=(-z+d, y+c, x-c), \\
& (x, y, z) S_{2}=(y, z, x),
\end{aligned}
$$

and $o$ as initial vertex. The faces of $P_{2}(c, d)$ are helices, three meeting at each vertex. If $P_{2}(c, d)$ is geometrically chiral, $c d \neq 0$ and its symmetry group is generated by $S_{1}$ and $S_{2}=S_{2}^{\prime}$. Whenever $c d=0$, the polyhedron $P_{2}(c, d)$ is orientably regular and $S_{1}$ and $S_{2}$ generate the even subgroup of its symmetry group.

With generators $S_{1}^{\prime}$ and $S_{2}$ the special group of $P_{2}(c, d)$ has the following presentation

$$
[4,3]^{+}=\left\langle S_{1}^{\prime}, S_{2} \mid\left(S_{1}^{\prime}\right)^{4}=S_{2}^{3}=\left(S_{1}^{\prime} S_{2}\right)^{2}=\varepsilon\right\rangle .
$$

If $c=0$ then $S_{1}^{4}=\varepsilon$ and $P_{2}(0,1)$ is combinatorially isomorphic to a cube [11, Lemma 5.7]. When $c \neq 0, S_{1}^{4}$ is the translation by the vector $(0,4 c, 0)$.

We define

$$
\begin{aligned}
& t_{1}:=S_{2}^{-1} S_{1}^{4} S_{2}, \\
& t_{2}:=S_{1}^{4}, \\
& t_{3}:=S_{2} S_{1}^{4} S_{2}^{-1} .
\end{aligned}
$$

Using Lemma 3.3, with the rotation $S_{2}$ as $R$, we can easily see that $t_{1}, t_{2}$ and $t_{3}$ are translations by the vectors $(4 c, 0,0),(0,4 c, 0)$ and $(0,0,4 c)$, respectively. Furthermore, it follows from [11, Lemma 5.2] that, if $c d \neq 0$, they generate the translation subgroup $\mathcal{T}\left(P_{2}(c, d)\right)$ of the symmetry group of $P_{2}(c, d)$. Using Theorem 3.2 we derive the following theorem.

Theorem 5.1 The symmetry group of the polyhedron $P_{2}(c, d)$ for $c d \neq 0$ is determined by the relations $S_{2}^{3}=\left(S_{1} S_{2}\right)^{2}=\varepsilon$ together with 


$$
\begin{aligned}
S_{2} S_{1}^{4} S_{2}^{-1} S_{1}^{4} & =S_{1}^{4} S_{2} S_{1}^{4} S_{2}^{-1}, \\
S_{1}^{-1} S_{2} S_{1}^{4} S_{2}^{-1} S_{1} & =S_{2}^{-1} S_{1}^{-4} S_{2} .
\end{aligned}
$$

Proof First, note that relation (26) is equivalent to the commutativity of $t_{2}$ and $t_{3}$. Conjugating this relation by $S_{2}$ and $S_{2}^{-1}$, it follows that $t_{1}$ commutes with $t_{2}$ and $t_{3}$. Hence, relations (7) are equivalent to relation (26).

The definitions of $t_{1}, t_{2}, t_{3}$ imply that $S_{2}^{-1} t_{i} S_{2}=t_{i+2}$ where $i$ is taken mod 3 . Also by definition, $S_{1}^{-1} t_{2} S_{1}=t_{2}$ and $S_{1}^{-1} t_{1} S_{1}=S_{1}^{-1} S_{2}^{-1} S_{1}^{4} S_{2} S_{1}=S_{2} S_{1}^{4} S_{2}^{-1}=t_{3}$. Finally, $S_{1}^{-1} t_{3} S_{1}=t_{1}^{-1}$ is just relation (27). It follows that relations (8) are reduced to relation (27). Finally, since $t_{2}=S_{1}^{4}$, relation (6) is superfluous.

Corollary 5.2 Up to isomorphism there is only one abstract polyhedron with chiral realization in $\mathbb{E}^{3}$ of Schläfli type $\{\infty, 3\}$ with helical faces over squares.

Proof The symmetry groups of any two chiral polyhedra of Schläfli type $\{\infty, 3\}$ with helical faces over squares are isomorphic since they have the same presentation; in fact, the defining relations for the symmetry group of $P_{2}(c, d)$ (we recall $c \neq 0$ ) do not involve the parameters $c$ or $d$ (see Lemma 2.3).

When $c \neq 0$ we can rescale the polyhedron $P_{2}(c, d)$ to obtain the polyhedron $P_{2}(1, d / c)$, which as we shall see below in Theorem 5.3, is isomorphic to $P_{2}(1,0)$. Schulte proved in [11] that the latter is geometrically isomorphic to Grünbaum's apeirohedron $\{\infty, 3\}^{(b)}$ described in [8, Chap. 7E]. The polyhedron $\{\infty, 3\}^{(b)}$ is regular and has helical faces. Following [8, Fig. 7E1], we project the vertices and edges of the apeirohedron to a plane $\Pi$ orthogonal to the axis of one of the helices resulting in the picture in Fig. 1. The three helices through any vertex of the apeirohedron will project onto a unit square containing that vertex, and two ("vertical" and "horizontal") zigzags of edges through the vertex. The edges of any helix that projects to a square can be drawn as diagonals of faces of cubes arranged in a stack over the square as shown in Fig. 2. Observe that the edges of the helices that do not project to a square in $\Pi$, project to edges connecting vertices of two different squares in Fig. 1

Fig. 1 Projection of the polyhedron $\{\infty, 3\}\}^{(b)}$

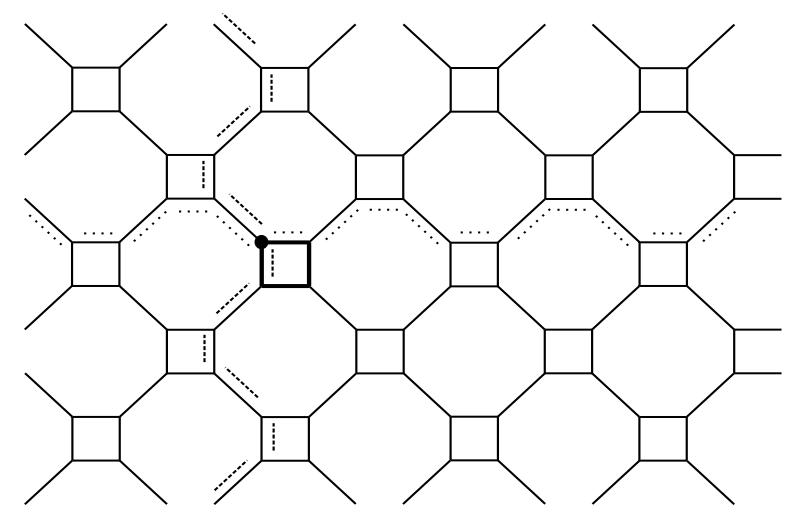


Fig. 2 Stacks of cubes and of square prisms

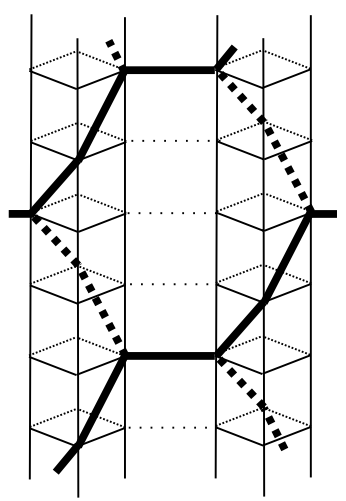

(a)

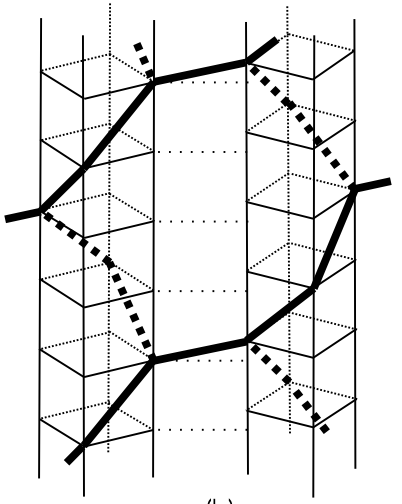

(b)

and are parallel to the plane of projection. We shall refer to these edges as bridges and note that they correspond to the horizontal edges between stacks of cubes over the squares (see also Fig. 2).

Theorem 5.3 Any polyhedron with discrete chiral realization in Euclidean 3-space of Schläfli type $\{\infty, 3\}$ and helical faces over squares is combinatorially isomorphic to $\{\infty, 3\}^{(b)}$, and hence, combinatorially regular.

Proof Corollary 5.2 implies that the polyhedra $P_{2}(c, d)$ for $c d \neq 0$ are all isomorphic. Note that the even subgroup of $P_{2}(1,0)$ coincides with the symmetry group of $P_{2}(c, d)$ for $c d \neq 0$. Lemma 2.2 now implies that the polyhedra $P_{2}(c, d)$ for $c \neq 0$ are all isomorphic.

The automorphism group of the apeirohedron $\{\infty, 3\}^{(b)}$ is the quotient of the Coxeter group $[\infty, 3]=\left\langle\rho_{0}, \rho_{1}, \rho_{2}\right\rangle$, determined by the single extra relation

$$
\left(\rho_{0} \rho_{1}\right)^{4}\left(\rho_{0} \rho_{1} \rho_{2}\right)^{3}=\left(\rho_{0} \rho_{1} \rho_{2}\right)^{3}\left(\rho_{0} \rho_{1}\right)^{4}
$$

[8, Theorem 7E22]. Multiplying the above relation by $\rho_{0}$ on the left and substituting $\rho_{i-1} \rho_{i}$ by $S_{i}(i=1,2)$ we conclude that the two defining relations (26) and (27) for the symmetry group of the polyhedron $P_{2}(c, d)$ can be replaced by a single relation $S_{1}^{-4} S_{2} S_{1}^{2} S_{2}^{-1}=S_{2} S_{1}^{2} S_{2}^{-1} S_{1}^{4}$.

We now return to the geometric considerations and show how, while preserving the combinatorial structure, $P_{2}(1,0)$ can continuously be transformed into $P_{2}(1, d)$ and $P_{2}(0,1)$ (recall that $P(c, d)$ is similar to $P(s c, s d)$ for $\left.s \neq 0\right)$. It is important to observe now that the axis of the base helix of $P_{2}(1, d)$ (the helix with vertex set $\left\{o S_{1}^{k}\right\}_{k \in \mathbb{Z}}$ ) is parallel to the $y$-axis. We now project $P_{2}(1, d)$ (including $d=0$ ) along this axis.

The helices over the squares in the projection of $P_{2}(1,0)=\{\infty, 3\}^{(b)}$ into the $x z$ plane (see Fig. 1) are drawn on stacks of cubes of volume 1 as shown in Fig. 2(a). Under the translation subgroup $4 \mathbb{Z}^{3}$ of the even subgroup of the symmetry group of $P_{2}(1,0)$, the stacks fall into two orbits with any two adjacent stacks (joined by a bridge) being in different orbits. 
Fig. 3 Projection of the polyhedra $P_{2}(1, d)$

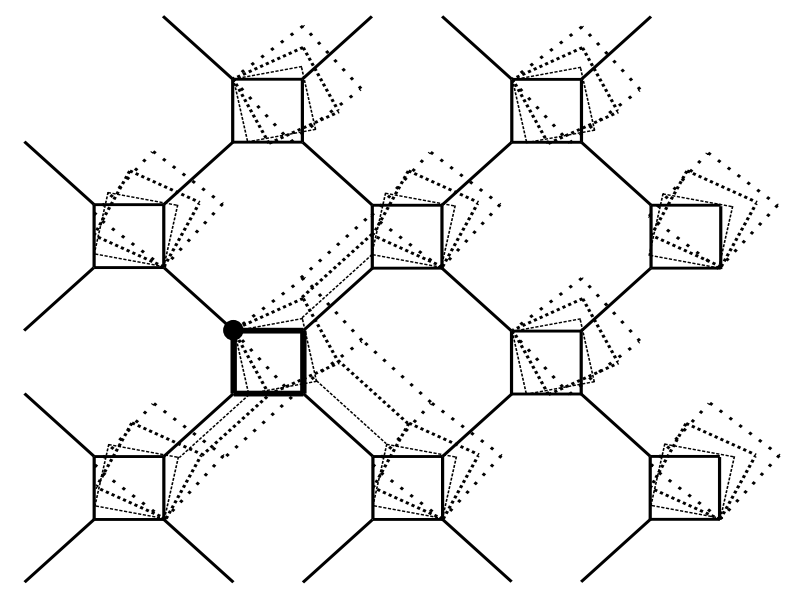

As $d$ changes, all vertices in the orbit of the origin $o$ under the translation subgroup will remain fixed. The $y$ coordinate of every vertex of the stacks of cubes containing translates of $o$ also remains unchanged and thus, the height of the cubes in the stacks is preserved. The $x$ - and $z$-coordinates of the vertex $o S_{2} S_{1} S_{2}^{-1}$ (the vertex adjacent to $o$ not in the same stack) are fixed, while its $y$-coordinate is changed by $d$. This induces a shifting with respect to the $x z$-plane of this stack, and also of all the stacks containing the translates of $o S_{2} S_{1} S_{2}^{-1}$. On the other hand, the $x$ - and $z$-coordinates of the remaining vertices increase or decrease by $d$ in such a way that the projection of $P_{2}(1, d)$ to the $x z$-plane is the one in Fig. 3. (Note that we give projections for several values of $d$ between 0 and 1.) It follows that the stacks of cubes (Fig. 2(a)) change into stacks of square prisms of height $c=1$ and base edge length $\sqrt{1+d^{2}}$ (Fig. 2(b)). In Fig. 3, we only include the bridges of $P_{2}(1,0)$ and the bridges of $P_{2}(1, d)$ emanating from the vertices of the stack containing the origin. Surprisingly, the length of the projections of bridges does not change although the length of the bridges does.

Note here that the full translation subgroup of $P_{2}(1,0)$ is $2 \Lambda_{(1,1,1)}$, which has index 2 in $4 \mathbb{Z}^{3}$. This implies that all the stacks over the squares in Fig. 1 are in the same orbit under the full translation subgroup of $P_{2}(1,0)$. However, as we mentioned earlier, the helices in the stacks over squares (and also the vertical and horizontal helices) of the polyhedron $P_{2}(1, d)$ for $d \neq 0$, split into two orbits. This leads to the six translation orbits of faces of $P_{2}(c, d)$ when $c d \neq 0$.

These geometric considerations can now be used to illustrate that $P_{2}(c, d)$ fails to be a geometric polyhedron whenever $d=k c$ and $k$ is an integer with $k \equiv 2(\bmod 4)$, as previously proved in [11, Lemma 5.5].

Let us now fix $d \neq 0$ and consider what happens when we decrease $c$ from 1 . More precisely, we shall consider $P_{2}(c, 1)$ and make $c$ approach 0 . First, note that every square in the projection of $P_{2}(c, 1)$ into the $x z$-plane is either a translate by a vector in $4 c \mathbb{Z}^{2}$ of the square with vertices $(0,0),(1,-c),(c+1,-c+1),(c, 1)$ if the corresponding helix is a translate of the base face, or of the square with vertices $(-c, c),(-c+1,2 c),(-2 c+1,2 c+1),(-2 c, c+1)$ if the corresponding helix is not a translate of the base face. This implies that, as $c$ approaches 0 , every square approaches the square with vertices $(0,0),(1,0),(1,1),(0,1)$. In addition, the height 
$c$ of the prisms in the stacks approaches 0 . Since similarly this happens with the projections on the $x y$-and $y z$-planes, it is not surprising that the limit, when $c$ goes to 0 , of the polyhedra $P_{2}(c, 1)$ is a cube with edge length 1 .

\subsection{Type $\{\infty, 3\}$ over Triangles}

We now turn our attention to the family consisting in polyhedra $P_{1}(a, b)$ of type $\{\infty, 3\}$ and helical faces over triangles. Using Wythoff's construction, the polyhedron $P_{1}(a, b)$ is built from the group generated by

$$
\begin{aligned}
& (x, y, z) S_{1}=(-z+b,-x+a, y), \\
& (x, y, z) S_{2}=(y, z, x),
\end{aligned}
$$

with $o$ as initial vertex. When the polyhedron $P_{1}(a, b)$ is geometrically chiral, $a \neq \pm b$ and its symmetry group is generated by $S_{1}$ and $S_{2}=S_{2}^{\prime}$. If $a= \pm b$ the polyhedron $P_{1}(a, b)$ is orientably regular and the symmetries $S_{1}$ and $S_{2}$ generate the even subgroup of its symmetry group.

The special group of $P_{1}(a, b)$ has the following presentation in terms of $S_{1}^{\prime}$ and $S_{2}$

$$
[3,3]^{+}=\left\langle S_{1}^{\prime}, S_{2} \mid\left(S_{1}^{\prime}\right)^{3}=S_{2}^{3}=\left(S_{1}^{\prime} S_{2}\right)^{2}=\varepsilon\right\rangle .
$$

If $a=b, S_{1}^{3}=\varepsilon$ and $P_{1}(1,1)$ is combinatorially isomorphic to a tetrahedron [11, Lemma 4.6]. Otherwise, if $a \neq b, S_{1}^{3}$ is the translation by the vector ( $b-a, a-$ $b, a-b)$.

We define $c=b-a$, and consider the following translations

$$
\begin{aligned}
& t_{1}:=S_{1}^{3}, \\
& t_{2}:=S_{2} S_{1}^{3} S_{2}^{-1}, \\
& t_{3}:=S_{2}^{-1} S_{1}^{3} S_{2} .
\end{aligned}
$$

Using Lemma 3.3, we can easily see that $t_{1}, t_{2}$ and $t_{3}$ are translations by the vectors $(c,-c,-c),(-c, c,-c)$ and $(-c,-c, c)$, respectively. Furthermore, it follows from [11, Lemma 4.2] that they generate the translation subgroup $\mathcal{T}\left(P_{1}(a, b)\right)$. Using Theorem 3.2, and bearing in mind that $P(a, b)$ is geometrically chiral only for $a \neq \pm b$, we derive the following theorem.

Theorem 5.4 The symmetry group of the polyhedron $P_{1}(a, b)$ when $a \neq \pm b$ is determined by the relations $S_{2}^{3}=\left(S_{1} S_{2}\right)^{2}=\varepsilon$ together with

$$
S_{1}^{3} S_{2} S_{1}^{3} S_{2}^{-1}=S_{2} S_{1}^{3} S_{2}^{-1} S_{1}^{3} .
$$

Proof First, note that relation (29) is equivalent to the commutativity of $t_{1}$ and $t_{2}$. Commutativity of $t_{3}$ with $t_{1}$ and $t_{2}$ is obtained conjugating (29) by $S_{2}^{-1}$ and $S_{2}$, respectively. Hence, relations (7) are equivalent to relation (29).

The definition of the translations $t_{i}$ implies that $S_{2}^{-1} t_{i} S_{2}=t_{i+2}$, where $i$ is taken mod 3. Furthermore, $S_{1}^{-1} t_{1} S_{1}=t_{1}$ and $S_{1}^{-1} t_{3} S_{1}=S_{1}^{-1} S_{2}^{-1} S_{1}^{3} S_{2} S_{1}=S_{2} S_{1}^{3} S_{2}^{-1}=t_{2}$. 
Note that $S_{1}^{-1} t_{2} S_{1}$ is the translation by the vector $(c, c, c)$ (we recall that $c=b-a$ ), which is $t_{1}^{-1} t_{3}^{-1} t_{2}^{-1}$. But, in fact, this last relation can be derived from the other relations stated in the theorem since

$$
\begin{aligned}
S_{1}^{-1} S_{2} S_{1}^{3} S_{2}^{-1} S_{1} & =S_{1}^{-2} S_{2}^{2} S_{1}^{2} S_{2}^{2} S_{1} \\
& =S_{1}^{-3} S_{2}^{-1} S_{1}^{-2} S_{2} S_{1}^{-2} S_{2}^{-1} \\
& =S_{1}^{-3} S_{2}^{-1} S_{1}^{-3} S_{2}^{-1} S_{1}^{-3} S_{2}^{-1} \\
& =t_{1}^{-1} t_{3}^{-1} t_{2}^{-1} .
\end{aligned}
$$

It follows that (29) implies relations (8). Finally, since $t_{1}=S_{1}^{3}$, relation (6) is superfluous.

Since the defining relations for the symmetry group of $P_{1}(a, b)$ (we recall $c \neq 0$ ) given in Theorem 5.4 do not involve the parameters $a$ or $b$, Lemma 2.3 implies

Corollary 5.5 Up to isomorphism there is only one abstract polyhedron with chiral realization in $\mathbb{E}^{3}$, Schläfli type $\{\infty, 3\}$ with helical faces over triangles.

In [8, Chap. 7E], McMullen and Schulte define Grünbaum's regular apeirohedron $\{\infty, 3\}^{(a)}$ as the Petrial of $\{\infty, 3\}^{(b)}$. That is, the two apeirohedra have the same vertex and edge set and, hence, the same edge length of $\sqrt{2}$, but the faces of $\{\infty, 3\}^{(a)}$ are Petrie polygons of $\{\infty, 3\}^{(b)}$ (Polygons whose two consecutive edges, but not three, belong to the same face.) Furthermore, Schulte proves in [11] that $\{\infty, 3\}^{(a)}$ is isomorphic to the regular polyhedron $P_{1}(1,-1)$. Using the projection of $\{\infty, 3\}^{(b)}$ in Fig. 1, we note that the helical faces of $\{\infty, 3\}^{(a)}$ can also be seen to project into zigzags which follow paths twice along a square and once along an octagon. It will be convenient to visualize this apeirohedron using a different projection described below.

The apeirohedron $\{\infty, 3\}^{(b)}$ has three orbits of (helical) faces under the translation subgroup, and every vertex belongs to one helix in each orbit. Note that there are 4 different orbits of helices under the translation subgroup of $P_{1}(a, b)$ for every $a \neq b$, and thus under the translation subgroup of $\{\infty, 3\}^{(a)}=P_{1}(1,-1)$ (see [11, Lemma 4.8]), and each vertex belongs to precisely three faces, one in each orbit. If we now project vertices and edges of $\{\infty, 3\}^{(a)}$ along the axis of a helix, vertices on that helix and all its translates, which we shall refer to as vertical helices, will project into vertices of triangles as indicated in Fig. 4. The edges of any vertical helix can be seen as diagonals of rectangular faces of a stack of triangular prisms over a triangle in the projection.

The vertices of $P_{1}(a, b)$, which do not project into vertices of triangles, lie on lines parallel to the axes of the vertical helices. These lines project into isolated points equidistant from the closest three triangles. The edges of the apeirohedron that do not project into the edges of the triangles form bridges that project into edges joining the isolated points with the (closest) triangles. The three bridges whose projections meet at one of the isolated points lie in planes parallel to the projection plane. Moreover, 


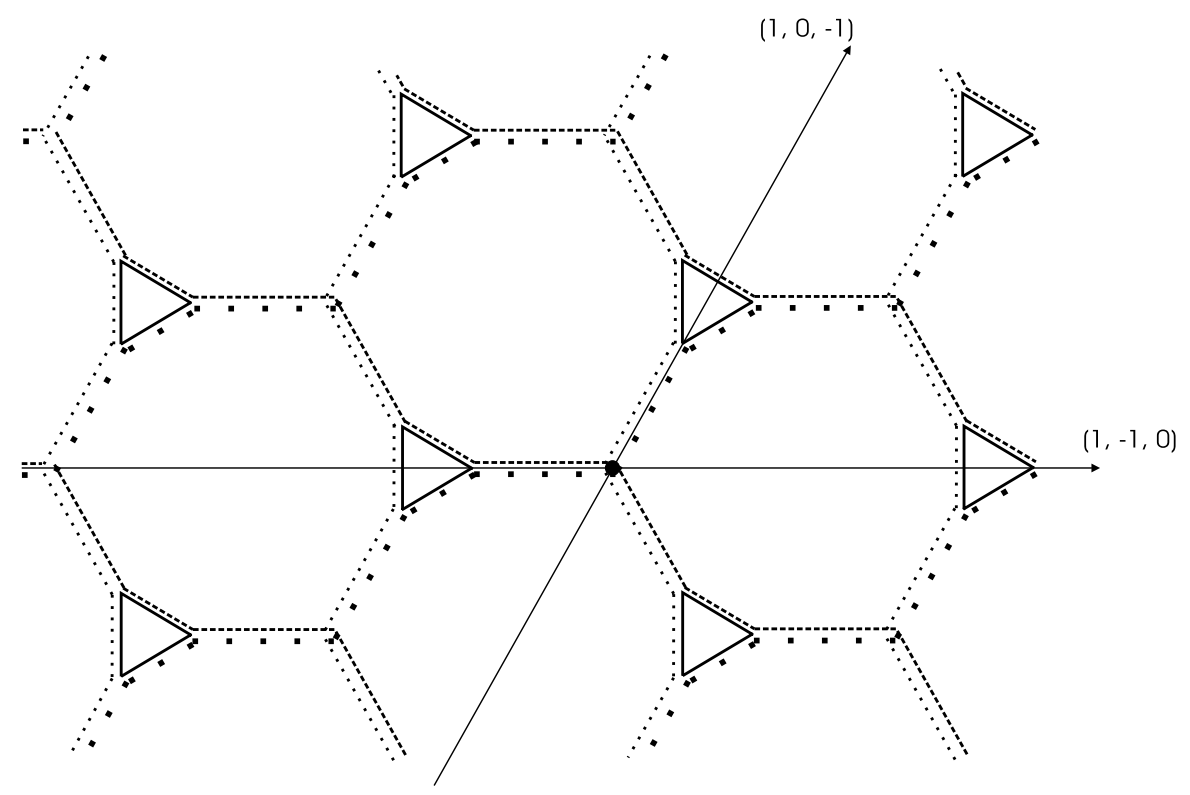

Fig. 4 Projection of the polyhedron $\{\infty, 3\}^{(a)}$

each bridge is parallel to the projection plane. This implies that all the vertices of the apeirohedron are arranged on planes which are parallel to the projection plane, with a fixed distance $2 / \sqrt{3}$ between consecutive planes, corresponding to the height of a triangular prism in a stack. The helices containing bridges as edges project into the zigzags indicated in Fig. 4.

Using Corollary 5.5 and Lemma 2.2, we now see that

Theorem 5.6 For every $a$ and every $b \neq a$, the polyhedron $P_{1}(a, b)$ is combinatorially isomorphic to $\{\infty, 3\}^{(a)}$, and hence, combinatorially regular.

The automorphism group of the apeirohedron $\{\infty, 3\}^{(a)}$ is the quotient of the Coxeter group $[\infty, 3]=\left\langle\rho_{0}, \rho_{1}, \rho_{2}\right\rangle$, determined by the single extra relation

$$
\left(\rho_{0} \rho_{1}\right)^{3}\left(\rho_{0} \rho_{1} \rho_{2}\right)^{4}=\left(\rho_{0} \rho_{1} \rho_{2}\right)^{4}\left(\rho_{0} \rho_{1}\right)^{3},
$$

[8, Theorem 7E22]. By replacing $\rho_{0} \rho_{1}$ by $S_{1}$ and $\rho_{1} \rho_{2}$ by $S_{2}$ in the previous equation, it can easily be seen that the defining relation (29) for the symmetry group of the polyhedron $P_{1}(a, b)$ may be replaced by a single relation $\left(S_{1}^{2} S_{2}^{2}\right)^{2} S_{1}^{3}=S_{1}^{3}\left(S_{1}^{2} S_{2}^{2}\right)^{2}$.

We now examine how the polyhedron $P_{1}(a, b)$ transforms when the parameters $a$ and $b$ are modified.

First, note that if $a \neq b$, the polyhedron $P_{1}(a, b)$ is similar to $P_{1}(d, d-2)$ with $d=2 a /(a-b)$. We shall see how the polyhedron $P_{1}(1,-1)=\{\infty, 3\}^{(a)}$ transforms into the polyhedron $P_{1}(a, a-2)$ while preserving its combinatorial structure. To see this, note that there is a translation class of helices of $P_{1}(a, a-2)$, which we will refer to as vertical helices, containing no helix through $o$. The vertex set of one of these 


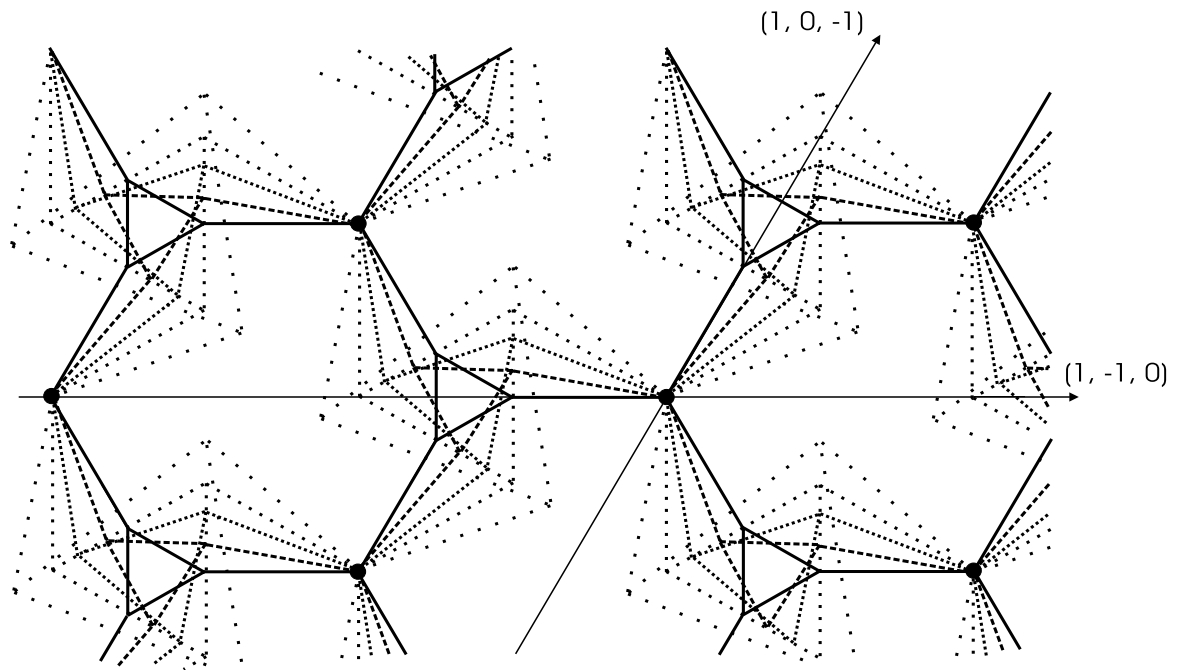

Fig. 5 Projection of the polyhedra $P_{1}(a, a-2)$

helices, according to [11, Lemma 4.8], is $\left\{o S_{1}^{k} S_{2}^{-1} S_{1} S_{2} \mid k \in \mathbb{Z}\right\}$. It follows (going three steps along this helix) that the axes of helices in this class are all in the direction of the vector $(1,1,1)$. This allows us to project $P_{1}(a, a-2)$ to the plane through $o$, perpendicular to the axes of the vertical helices, referred to as horizontal plane. In other words, for all values of $a$, the vertical helices will project onto triangles in the horizontal plane. Hence, again the edges of $P_{1}(a, a-2)$ can be seen as bridges or diagonals of rectangular faces of triangular prisms arranged in stacks. The translation subgroup (generated by the translations in (28) with $c=-2)$ of $P_{1}(a, a-2)$ coincides with the translation subgroup of $P_{1}(1,-1)$. Since the translation vector given by three steps along any helix is preserved, it follows that, as $a$ changes, the height of the triangular prisms in the stacks remains unchanged.

To see how the stacks change when $a$ changes, first observe that all the vertices that project into isolated vertices are fixed. The vertices that belong to stacks of triangular prisms move vertically (along lines of projection) for $2(a-1) / 3$, and horizontally (parallel to the plane of projection) as indicated in Fig. 5 for several values of $a$. Observe that the axes of the helices on stacks remain unchanged; however, the bridges are no longer parallel to the horizontal plane. Once again, this illustrates that the polyhedra $P_{1}(a, b)$ with $a \neq b$ are all combinatorially isomorphic.

Similar considerations to those used in transforming $P_{2}(c, d)$ will show how the polyhedron $P_{1}(a, a-\delta)$ approaches a regular tetrahedron when $\delta$ approaches 0 .

\subsection{Type $\{\infty, 4\}$}

Finally, we examine the family consisting of polyhedra $P_{3}(c, d)$ of type $\{\infty, 4\}$ with helical faces over triangles. As we shall see below, one regular member of this family is non-orientably regular and consequently the chiral members of the family are chiral realizations of its double cover. 
As before, we build the geometric polyhedron $P_{3}(c, d)$ from the group generated by

$$
\begin{aligned}
& (x, y, z) S_{1}=(z-d, x-c, y+c), \\
& (x, y, z) S_{2}=(z, y,-x)
\end{aligned}
$$

using Wythoff's construction with $o$ as initial vertex.

The special group of $P_{3}(c, d)$ can be presented in terms of $S_{1}^{\prime}$ and $S_{2}=S_{2}^{\prime}$ as follows

$$
[3,4]^{+}=\left\langle S_{1}^{\prime}, S_{2} \mid\left(S_{1}^{\prime}\right)^{3}=S_{2}^{4}=\left(S_{1}^{\prime} S_{2}\right)^{2}=\varepsilon\right\rangle .
$$

If $d=0$ then $S_{1}^{3}=\varepsilon$ and $P_{3}(1,0)$ is combinatorially isomorphic to a regular octahedron [11, Lemma 6.5], otherwise $S_{1}^{3}$ is the translation by the vector $(-d,-d,-d)$.

Now we define the translations

$$
\begin{aligned}
& t_{1}:=S_{2} S_{1}^{3} S_{2}^{-1}, \\
& t_{2}:=S_{1}^{3}, \\
& t_{3}:=S_{2}^{-1} S_{1}^{3} S_{2} .
\end{aligned}
$$

The definition of $S_{1}$ and Lemma 3.3 imply that $t_{1}, t_{2}$ and $t_{3}$ are translations by the vectors $(d,-d,-d),(-d,-d,-d)$ and $(-d,-d, d)$, respectively. Furthermore, it follows from [11, Lemma 6.1] that they generate the translation subgroup $\mathcal{T}\left(P_{3}(c, d)\right.$ ) of the symmetry group of $P_{3}(c, d)$.

Using Theorem 3.2 and observing that according to [11, Lemma 6.5] $P_{3}(0,1)$ is non-orientably regular, we derive the following theorem.

Theorem 5.7 The symmetry group of the polyhedron $P_{3}(c, d)$ for $d \neq 0$ is determined by the relations $S_{2}^{4}=\left(S_{1} S_{2}\right)^{2}=\varepsilon$ together with

$$
\begin{aligned}
S_{2}^{-1} S_{1}^{3} S_{2} S_{1}^{3} & =S_{1}^{3} S_{2}^{-1} S_{1}^{3} S_{2}, \\
S_{2} S_{1}^{3} S_{2}^{2} S_{1}^{3} S_{2} & =S_{2}^{-1} S_{1}^{3} S_{2}^{2} S_{1}^{3} S_{2}^{-1}, \\
S_{1}^{-1} S_{2} S_{1}^{3} S_{2}^{-1} S_{1} & =S_{2} S_{1}^{-3} S_{2}^{-1} S_{1}^{3} S_{2}^{-1} S_{1}^{-3} S_{2}, \\
S_{2}^{2} S_{1}^{3} S_{2}^{2} & =S_{1}^{-1} S_{2} S_{1}^{-3} S_{2}^{-1} S_{1} .
\end{aligned}
$$

Proof First, note that relation (30) is equivalent to the commutativity of $t_{2}$ and $t_{3}$. Conjugating this relation by $S_{2}^{-1}$ it follows that $t_{1}$ commutes with $t_{2}$. Furthermore, relation (31) is equivalent to the commutativity of $t_{1}$ and $t_{3}$. Hence, relations (7) can be replaced by (30) and (31).

By definition, $S_{1}^{-1} t_{2} S_{1}=t_{2}$ and $S_{1}^{-1} t_{3} S_{1}=S_{1}^{-1} S_{2}^{-1} S_{1}^{3} S_{2} S_{1}=S_{2} S_{1}^{3} S_{2}^{-1}=t_{1}$. Finally, $S_{1}^{-1} t_{1} S_{1}$ is the translation by the vector $(-d, d,-d)$, which is the translation $t_{1}^{-1} t_{2} t_{3}^{-1}$ implying relation (32).

The definition of $t_{1}, t_{2}$ and $t_{3}$ imply the relations $S_{2}^{-1} t_{i} S_{2}=t_{i+1}$ for $i=1,2$. Note that $S_{2}^{-1} t_{3} S_{2}$ corresponds to the translation by the vector $(d,-d, d)$, which is the 
Fig. 6 Projection of the polyhedron $\{\infty, 4\}$., 3

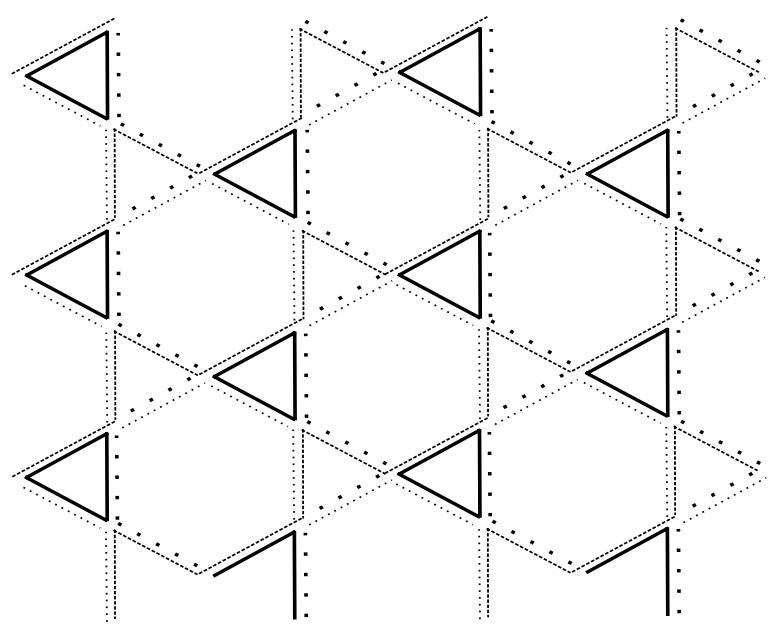

translation $t_{1} t_{2}^{-1} t_{3}$, implying relation (33). It follows that relations (8) can be replaced by relations (32) and (33). Finally, since $t_{2}=S_{1}^{3}$, relation (6) is superfluous.

Corollary 5.8 Up to isomorphism there is only one abstract polyhedron with chiral realization in $\mathbb{E}^{3}$ and Schläfli type $\{\infty, 4\}$.

Proof The defining relations for the symmetry group of $P_{3}(c, d)$ (we recall $d \neq 0$ ) do not involve the parameters $c$ or $d$. Hence the even subgroups of any two such automorphism groups are isomorphic implying that all such polyhedra are combinatorially isomorphic.

Schulte proved in [11, Lemma 6.5] that $P_{3}(0,1)$ is geometrically isomorphic to Dress's regular apeirohedron denoted by $\{\infty, 4\}_{, * 3}$ in [8, Sect. 7E] and [9], and whose automorphism group is the quotient of the Coxeter group $[\infty, 4]$ determined by the extra relation $\left(\rho_{2}\left(\rho_{1} \rho_{0}\right)^{2}\right)^{3}=\varepsilon$ (see [11, Theorem 7E22]). Note that this polyhedron is non-orientably regular.

We now use the ideas developed above of projecting an infinite polyhedron with helical faces along the axis of one of its helices. Note first that $\{\infty, 4\}_{, * 3}$ has four orbits of faces under the full translation subgroup. The helices in one of the orbits will project into the (solid line) triangles in Fig. 6 and the helices in the other three orbits will project into zigzags indicated in the same figure (here we projected with respect to the axis of the base helix). Observe that no edges of $\{\infty, 4\}\}_{, * 3}$ are parallel to the plane of projection.

Since $P_{3}(0,1)$ is non-orientably regular, it does not have a chiral realization. However, the authomorphism group of $P_{3}(0,1)$ coincides with its even subgroup which is isomorphic to the symmetry subgroup of $P_{3}(c, d)$ for $c d \neq 0$. This implies that $P_{3}(c, d)$ for $c d \neq 0$ is isomorphic to the orientably regular double cover of $P_{3}(0,1)$. Using Corollary 5.8, we see that 
Fig. 7 Projection of a geometrically chiral polyhedron $P_{3}(c, d)$

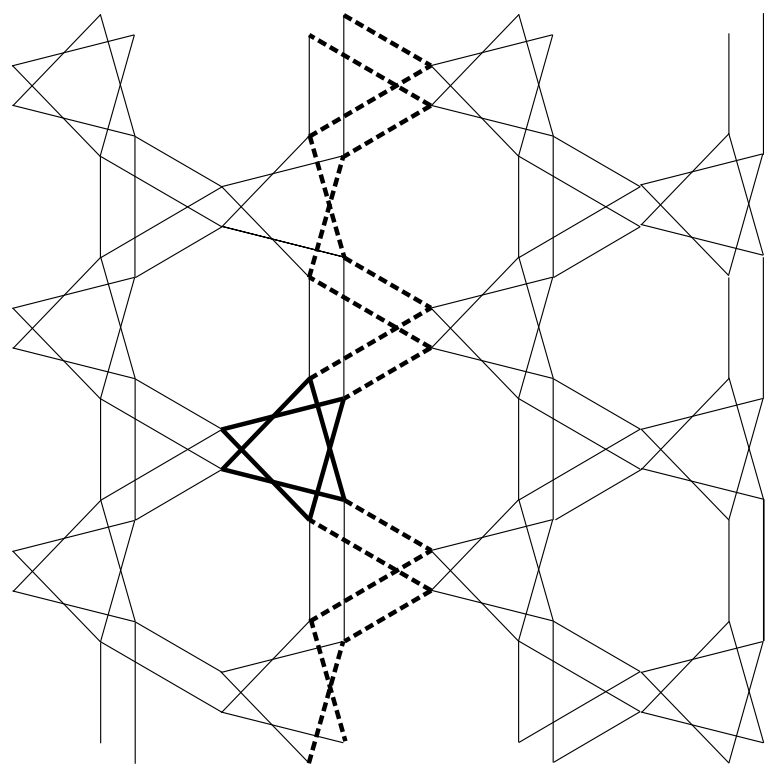

Theorem 5.9 For every $c \neq 0$, the polyhedron $P_{3}(c, 1)$ is combinatorially isomorphic to the orientably regular double cover of $\{\infty, 4\} ., 3$, and hence, is combinatorially regular.

Assuming $c \neq 0$, for each helical face of $P_{3}(c, 1)$ there is exactly one other helix, which we shall call its $t$ win, sharing the same axis. In fact, each twin pair corresponds to a single helix of $P_{3}(0,1)$ under the quotient determined by the double cover. Every helix and its twin belong to two different orbits under the full translation subgroup. Fixing a non-zero value of $c$ we project $P_{3}(c, 1)$ along the axis $L$ of the base helix. The base helix and its twin project to two triangles with cocyclic vertices indicated with thick solid lines in Fig. 7. There are three helical faces through $o$ distinct from the base helix. The projection of one such helix and its twin is shown in thick dashed lines in Fig. 7. The other two pairs of helices have similar projections.

With these observations it is not difficult to see how the polyhedron $P_{3}(c, 1)$ transforms when the parameter $c \neq 0$ is modified. One can also see that $P_{3}(c, d)$ fails to be a geometric polyhedron whenever $c / d$ is a non-zero integer as proved in [11, Lemma 6.3]. Furthermore, similar considerations to those of $P_{2}(c, d)$ will show how the polyhedron $P_{3}(1, d)$ approaches a regular octahedron when $d$ approaches 0 .

\section{Concluding Remarks}

There has been little research done on realizations of chiral polytopes and on chiral realizations of regular polytopes (see [14, Sect. 5]). Among other things the present paper contributes the following two results. 
Corollary 6.1 The only chiral polyhedra with discrete (chiral) realizations in Euclidean 3-space are the polyhedra in the families $P(a, b)$ for $a, b$ coprime integers with $a \neq \pm b$, and the polyhedra $Q(c, d)$ and $Q_{1}(c, d)$ for $c, d$ coprime integers with $c d \neq 0$.

Corollary 6.2 The only regular polyhedra with discrete chiral realizations in Euclidean 3-space are $\{\infty, 3\}^{(a)},\{\infty, 3\}^{(b)}$ and the orientably regular double cover of $\{\infty, 4\}_{\cdot, * 3}$.

The first corollary is implied by [10] and Theorems 4.1 and 4.3, while the second follows directly from Theorems 5.3, 5.6 and 5.9.

\section{References}

1. Conder, M.: List of regular maps and hypermaps and trivalent symmetric graphs. http://www.m.math. auckland.ac.nz/ conder/

2. Conder, M., Dobcsányi, P.: Determination of all regular maps of small genus. J. Comb. Theory, Ser. B 81, 224-242 (2001)

3. Coxeter, H.S.M.: Regular skew polyhedra in 3 and 4 dimensions and their topological analogous. Proc. Lond. Math. Soc. 43, 33-62 (1937)

4. Coxeter, H.S.M., Moser, W.O.J.: Generators and Relations for Discrete Groups, 4th edn. Springer, Berlin (1980)

5. Dress, A.W.M.: A combinatorial theory of Grünbaum's new regular polyhedra, I: Grünbaum's new regular polyhedra and their automorphism group. Aequ. Math. 23, 252-265 (1981)

6. Dress, A.W.M.: A combinatorial theory of Grünbaum's new regular polyhedra, II: complete enumeration. Aequ. Math. 29, 222-243 (1985)

7. Grünbaum, B.: Regular polyhedra—old and new. Aequ. Math. 16, 1-20 (1977)

8. McMullen, P., Schulte, E.: Abstract Regular Polytopes. Encyclopedia of Mathematics and Its Applications, vol. 92. Cambridge University Press, Cambridge (2002)

9. McMullen, P., Schulte, E.: Regular polytopes in ordinary space. Discrete Comput. Geom. 17, 449-478 (1997)

10. Schulte, E.: Chiral polyhedra in ordinary space, I. Discrete Comput. Geom. 32, 55-99 (2004)

11. Schulte, E.: Chiral polyhedra in ordinary space, II. Discrete Comput. Geom. 34, 181-229 (2005)

12. Schulte, E., Weiss, A.I.: Chiral polytopes. In: Gritzmann, P., Sturmfels, B. (eds.) Applied Geometry and Discrete Mathematics (The "Victor Klee Festschrift"). DIMACS Ser. Discrete Math. Theoret. Comput. Sci., vol. 4, pp. 493-516 (1991)

13. Schulte, E., Weiss, A.I.: Chirality and projective linear groups. Discrete Math. 131, 221-261 (1994)

14. Schulte, E., Weiss, A.I.: Problems on polytopes, their groups, and realizations. Period. Math. Hung. 53, 231-255 (2006) 